\title{
ETV2 regulates PARP-1 binding protein to induce ER stress-mediated death in tuberin-deficient cells
}

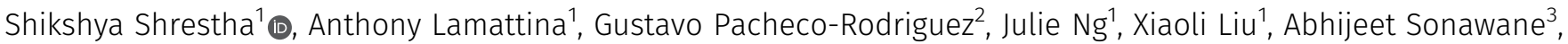

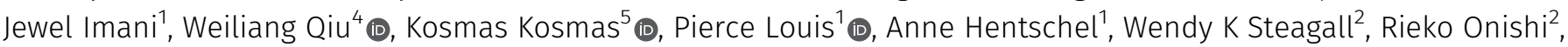 \\ Helen Christou ${ }^{5}$, Elizabeth P Henske ${ }^{1}$, Kimberly Glass ${ }^{4}$, Mark A Perrella ${ }^{1,5}$ @i, Joel Moss ${ }^{2}$, Kelan Tantisira ${ }^{4,6}$, \\ Souheil El-Chemaly ${ }^{1}$ (1)
}

Lymphangioleiomyomatosis (LAM) is a rare progressive disease, characterized by mutations in the tuberous sclerosis complex genes (TSC1 or TSC2) and hyperactivation of mechanistic target of rapamycin complex 1 (mTORC1). Here, we report that E26 transformation-specific (ETS) variant transcription factor 2 (ETV2) is a critical regulator of Tsc2-deficient cell survival. ETV2 nuclear localization in Tsc2-deficient cells is mTORC1-independent and is enhanced by spleen tyrosine kinase (Syk) inhibition. In the nucleus, ETV2 transcriptionally regulates poly(ADP-ribose) polymerase 1 binding protein (PARPBP) mRNA and protein expression, partially reversing the observed down-regulation of PARPBP expression induced by mTORC1 blockade during treatment with both Syk and mTORC1 inhibitors. In addition, silencing Etv2 or Parpbp in Tsc2-deficient cells induced ER stress and increased cell death in vitro and in vivo. We also found ETV2 expression in human cells with loss of heterozygosity for TSC2, lending support to the translational relevance of our findings. In conclusion, we report a novel ETV2 signaling axis unique to Syk inhibition that promotes a cytocidal response in Tsc2-deficient cells and therefore maybe a potential alternative therapeutic target in LAM.

DOI 10.26508/Isa.202201369 | Received 10 January 2022 | Revised 21 January 2022 | Accepted 21 January 2022 | Published online 18 February 2022

\section{Introduction}

Lymphangioleiomyomatosis (LAM) is a rare, multisystem disease associated with smooth muscle-like "LAM cells" over-proliferating in lungs, kidneys, and lymphatics. LAM cells have inactivating mutations in either tuberous sclerosis complex 1 or 2 (TSC1 or TSC2) genes, leading to hyperactivation of the mechanistic/mammalian target of rapamycin complex 1 (mTORC1) signaling (Carsillo et al, 2000; Cheadle et al, 2000; Goncharova et al, 2002; Crino et al, 2006; Johnson et al, 2016; Rosset et al, 2017). A pivotal clinical trial has led to the approval of the allosteric mTORC1 inhibitor sirolimus (rapamycin) by the Food and Drug Administration for use in LAM (McCormack et al, 2011). However, because of the cytostatic nature, continuous treatment with rapamycin is required to maintain lung function stability (Yao et al, 2014). Therefore, there is a need for a better understanding of tuberin-deficient cell death mechanisms, which could potentially lead to novel therapies.

We have previously shown that there is increased expression and activation of spleen tyrosine kinase (Syk) in both Tsc2-deficient cells and LAM lung nodules (Cui et al, 2017). Similar to rapamycin treatment, R406, a Syk inhibitor (Sykl) had antiproliferative effects in Tsc2-deficient cells in vitro and in vivo (Cui et al, 2017). Sykdependent regulation of mTOR signaling has been previously documented in B-cell lymphoma (Leseux et al, 2006; Fruchon et al, 2012) and acute myeloid leukemia (Carnevale et al, 2013). We demonstrated that mTORC1 inhibition altered Syk expression and activity, suggesting a feedback loop between the two pathways in Tsc2-deficient cells (Cui et al, 2017). Here, we sought to investigate regulatory pathways of Syk inhibition that are independent of its cross-talk with mTORC1 signaling in Tsc2-deficient cells and potentially identify target(s) of Sykl that can have cytotoxic effects on Tsc2-deficient cells.

We performed an expression array of Syk and mTORC1 inhibition to identify unique regulatory mechanism(s) between the two signaling pathways. We then used Passing Attributes between Networks for Data Assimilation (PANDA) (Glass et al, 2013) to reconstruct treatmentspecific transcription factor regulatory networks in Tsc2-deficient cells. Using these networks, we identified E26 transformation-specific (ETS) variant transcription factor 2 (ETV2) that targets a unique set of genes in

\footnotetext{
'Division of Pulmonary and Critical Care Medicine, Brigham and Women's Hospital, Harvard Medical School, Boston, MA, USA ${ }^{2}$ Division of Intramural Research, Pulmonary Branch, National Heart, Lung and Blood Institute (NHLBI), National Institutes of Health (NIH), Bethesda, MD, USA ${ }^{3}$ Department of Cardiovascular Medicine, Brigham and Women's Hospital, Harvard Medical School Boston, MA, USA ${ }^{4}$ Channing Division of Network Medicine, Brigham and Women's Hospital, Harvard Medical School, Boston, MA, USA ${ }^{5}$ Department of Pediatric Newborn Medicine, Brigham and Women's Hospital, Harvard Medical School, Boston, MA, USA ${ }^{6}$ Division of Pediatric Pulmonary and Critical Care Medicine, University of California San Diego, La Jolla, CA, USA
} 
the Sykl network independent of mTORC1 inhibition and is a critical regulator of Tsc2-deficient cell survival.

ETV2 is a member of the ETS family of transcription factors, which plays a key role in the development of hematopoietic and endothelial lineages (Liu et al, 2015; Garry, 2016). Etv2 deficiency is associated with a complete blockage in blood and endothelial cell formation (Garry, 2016; Oliver \& Srinivasan, 2010). In zebrafish, direct binding of ETV2 to the Lyve1 and Vegfr-3 promoter/enhancer results in the transcriptional regulation of those lymphatic markers (Davis et $\mathrm{al}$, 2018). The role of ETV2 in lymphangiogenesis potentially has functional implications in LAM as there are lymphatic manifestations in LAM, including increased expression of VEGF-D in the serum of LAM patients (Seyama et al, 2006; Glasgow et al, 2008; Young et al, 2010). Studies also have demonstrated the potential lymphatic origin of LAM or Tsc2-deficient cells (Davis et al, 2013; Yue et al, 2016). Recently, studies have demonstrated ETV2 mRNA expression and amplification in various tumor specimens, including glioblastoma and adrenocortical carcinoma (Li et al, 2018; Zhao et al, 2018). The activation of ETV2 in tumor-associated endothelial cells was shown to contribute to tumor angiogenesis (Kabir et al, 2018).

We demonstrated that ETV2 differentially regulates a unique set of genes in the Sykl treatment network, including poly(ADP-ribose) polymerase 1 (PARP1) binding protein (PARPBP). PARPBP, also known as PARI or C12orf48, is a replisome-associated protein that plays important roles during replication stress and DNA repair through its interaction with PARP-1, PCNA, and RAD51 (Varisli, 2013; Burkovics et al, 2016; Nicolae et al, 2019). PARPBP overexpression is associated with hyperproliferation in pancreatic cancers, hepatocellular carcinoma, and myeloid leukemia (Piao et al, 2011; O'Connor et al, 2013; Nicolae et al, 2019; Yu et al, 2019). PARP-1 inhibitors are promising antineoplastic agents (Malyuchenko et al, 2015) and have been shown to selectively inhibit proliferation and induce apoptosis in Tsc2-deficient cells (Malyuchenko et al, 2015). However, the role of PARPBP in LAM or TSC2-deficient cells and its regulation are largely unknown. Our data showed that ETV2 transcriptionally regulates PARPBP. We further observed increased ER stress and increased Tsc2-deficient cell death when Etv2 or Parpbp was silenced. Hence, targeting ETV2, with its potential for cytocidal cellular responses in Tsc2-deficient cells, might offer a therapeutic advantage in LAM over rapalogs alone, which primarily act as cytostatic drugs.

\section{Results}

\section{Gene expression profiling in rapamycin- versus Sykl-treated Tsc2-deficient cells}

To discern the effects on gene expression of mTORC1 and Syk inhibition in TSC2-deficient cells, we ran Rat Gene 2.0 ST microarrays with ELT3-V cells treated with DMSO, Sykl, or rapamycin for $24 \mathrm{~h}$. A heatmap generated using the top 485 most differentially expressed genes in each treatment condition compared with the control (DMSO) showed four distinct clusters (Fig 1A). We validated the gene expression data for the top 8 differentially expressed genes from each cluster using RT-qPCR (Fig S1A-D). The principal component analysis plot, based on PC1, revealed DMSO-treated replicates clustered very distinctly from both Sykl- and rapamycin-treated replicates, suggesting considerable changes in gene expression in the treatment groups compared with DMSO (Fig 1B). In contrast, only a minute separation in the clusters was observed between the two treatment groups, suggesting largely concordant changes in gene expression (Fig 1B).

The top cluster (Cluster 1, red) in the heatmap included 201 genes that were similarly down-regulated in both Sykl- and rapamycintreated cells compared with DMSO-treated cells (Fig 1A). Gene ontology (GO) enrichment analysis on these genes showed upregulation in biological processes that include oxidation-reduction and L-serine biosynthesis (Fig 1C). The second cluster (Cluster 2, green) included 107 genes that were down-regulated in both treatment groups compared with DMSO but demonstrated greater down-regulation with rapamycin than with Sykı (Fig 1A). These genes corresponded to 17 significantly up-regulated biological processes, most of which were associated with the cell cycle (Fig 1C). Only one gene (Mospd1) was down-regulated by SykI treatment, but not rapamycin, comprising the third cluster (Cluster 3, blue). RTqPCR validation however showed down-regulation of Mospd in both Sykl and rapamycin treatment groups compared with DMSO (Fig S1C). The fourth cluster (Cluster 4, pink) included 176 genes that were up-regulated in both treatment groups compared with DMSO and corresponded to a significant up-regulation of biological processes associated with cellular migration (Fig 1A and C).

Overall, differential expression data from the microarray indicated that Sykl and rapamycin treatments similarly impacted gene expression in Tsc2-deficient cells. A subset of genes, comprising Cluster 2, were observed to be more down-regulated with mTORC1 inhibition than with Syk inhibition.

\section{Network analysis reveals putative Sykl-specific regulation driven by the transcription factor Etv2}

To further understand potential gene regulation differences between SykI and rapamycin treatments, we used PANDA analysis to integrate information from treatment-specific gene expression and transcription factor binding motifs and construct transcriptional regulatory networks for the three treatment groups using pairwise comparisons between two groups at a time: Sykl versus DMSO, rapamycin versus DMSO, and SykI versus rapamycin. Edge weight differences for the top 10,000 (TF, gene) pairs with the largest absolute differences of edge weights between two networks Sykl and rapamycin were plotted (Fig 1D). Consistent with the finding from differential gene expression and pathway analyses, a high correlation between the edge weights for the two treatment groups was observed; however, we also found regulatory edges that were more strongly supported by either Sykl (red) or rapamycin (blue) treatment. Based on the top 10,000 (TF, gene) pairs, Sykl and rapamycin networks consisted of 54 unique TFs in either of the two networks, and most TFs belonged to the members of the E26 transformation-specific (Ets) family of TFs (Table 1). Ets variant 2 (ETV2) was among the top 20 TFs regulating the highest numbers of genes in each network. Because evidence suggested a potential lymphatic endothelial origin of LAM cells (Davis et al, 2013; Yue et al, 2016) and that ETV2 is critical for lymphangiogenesis (Davis et al, 2018), we further investigated the role of ETV2. 

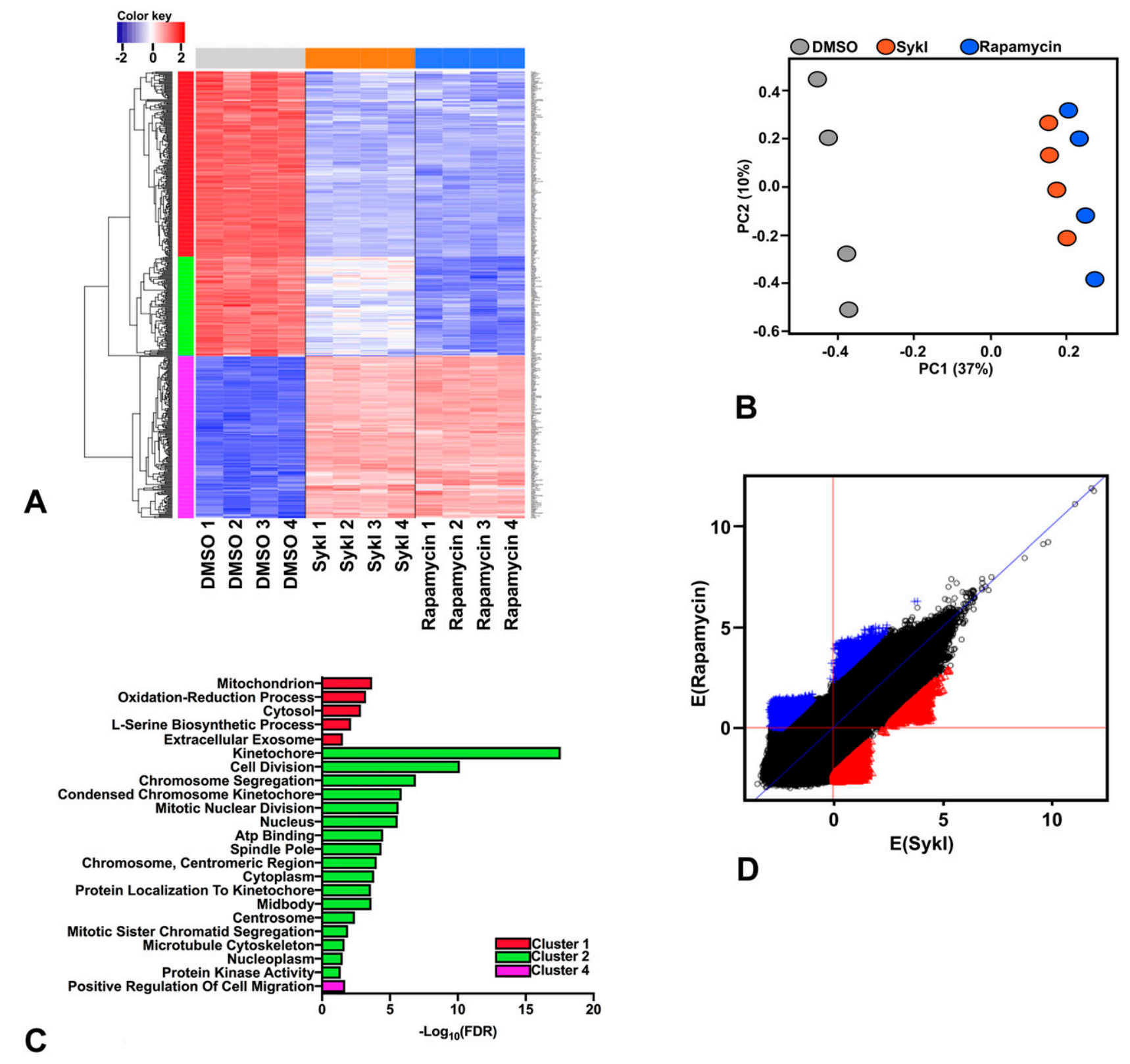

B

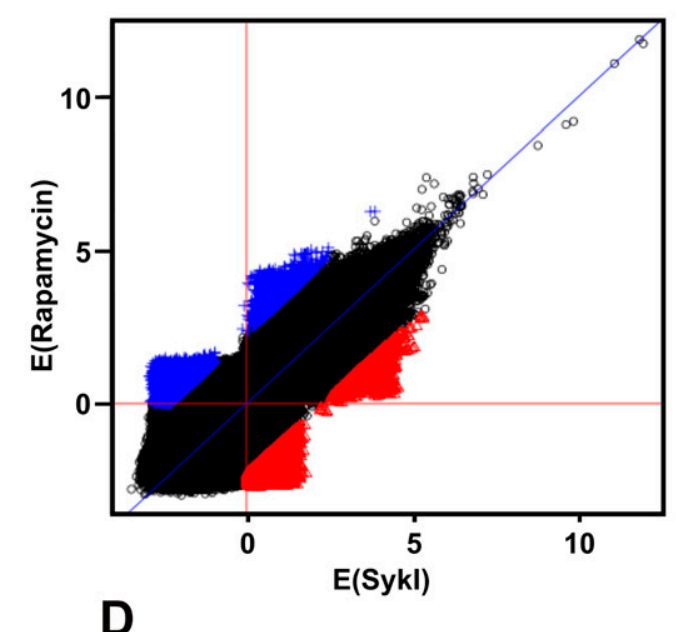

Figure 1. Gene expression profiling in rapamycin- versus Sykl-treated ELT3-V cells.

(A) TsC2-deficient Eker rat (ELT3-V) cells were treated with SykI (R406; $1 \mu \mathrm{M}$ ), rapamycin (20 nM), or DMSO for $24 \mathrm{~h}$. Data were obtained using Affymetrix Rat Gene $2.0 \mathrm{ST}$ GeneChip. The diagram represents 485 differentially expressed probes $(q<0.01)$ with at least twofold change between conditions. Each column represents a single experiment and each row a single gene. The colors are scaled so that red and blue indicate $z$-scores of $\geq 2$ or $\leq-2$, respectively, and white indicates a $z$-score of 0 (row-wise mean). Color blocks on top, gray, orange, and blue represent different treatment groups, DMSO, Sykl, and rapamycin, respectively. Color blocks on the left, red, green, blue, and pink represent four different clusters, Clusters 1-4. (B) Principal component analysis of samples treated with DMSO, rapamycin, or Sykl for 24 h. Principal component analysis was performed across all probes on the microarray and a bi-plot of PC1 against PC2 was plotted to show clustering of different treatment groups and condition replicates. $(\mathbf{A}, \mathbf{C})$ Gene ontology enrichment analysis for significantly up-regulated biological processes using genes in each cluster in the heatmap $(A)$. Gene ontology terms for Clusters 1, 2, and 4 are presented in red, green, and pink, respectively. (D) Scatter plot for the Sykl and rapamycin networks predicted by PANDA for the top 10,000 edges (TF, gene) in each of the networks models, rapamycin treatment, and Sykl treatment models. Each point in the graph represents a difference in edge weight connecting a transcription factor to a target gene between two networks. Red points represent the 10,000 (TF, gene) pairs having largest weight in Sykl network, and blue points represent (TF, gene) edges having the largest weight in rapamycin network.

\section{Sykl, but not rapamycin, treatment induced ETV2 translocation into cell nuclei}

To understand a potential role for ETV2 in mediating Sykldependent function in Tsc2-deficient cells, we investigated Etv2 gene and ETV2 protein expression in Tsc2-deficient ELT3-V cells treated with DMSO, Sykl, or rapamycin for $24 \mathrm{~h}$. No significant differences in both total mRNA and total protein expression were observed with either Sykl or rapamycin treatment compared with DMSO (Fig 2A-C). SYK has previously been shown to regulate the 
Table 1. TF with highest nDiff in Sykl and Rapa networks identified using top 10,000 (TF, genes) pairs.

\begin{tabular}{|c|c|c|c|c|c|c|c|}
\hline Gene & TF motif ID & TF family & nSykı & nRapa & nDiff & nOverlap & nRatio \\
\hline Elk4 & M0703 & ETS domain & 1,106 & 1,064 & 42 & 0 & 1.04 \\
\hline Elk3 & M0692 & ETS domain & 1,051 & 1,005 & 46 & 0 & 1.05 \\
\hline Gm5454 & M0710 & ETS domain & 1,039 & 972 & 67 & 0 & 1.07 \\
\hline Etv3 & M0689 & ETS domain & 916 & 862 & 54 & 0 & 1.06 \\
\hline Erfl & M0714 & ETS domain & 912 & 869 & 43 & 0 & 1.05 \\
\hline Elf4 & M0706 & ETS domain & 687 & 666 & 21 & 0 & 1.03 \\
\hline Etv6 & M0705 & ETS domain & 665 & 618 & 47 & 0 & 1.08 \\
\hline Elk1 & M6207 & ETS domain & 621 & 590 & 31 & 0 & 1.05 \\
\hline $1 d 4$ & M5571 & Basic helix-loop-helix & 346 & 356 & -10 & 0 & 0.97 \\
\hline Erf & M5398 & ETS domain & 327 & 327 & 0 & 0 & 1.00 \\
\hline Myod1 & M2299 & Basic helix-loop-helix & 297 & 308 & -11 & 0 & 0.96 \\
\hline Tcfl3 & M0184 & Basic helix-loop-helix & 241 & 242 & -1 & 0 & 1.00 \\
\hline Myog & M2300 & Basic helix-loop-helix & 200 & 201 & -1 & 0 & 1.00 \\
\hline Ehf & M0696 & ETS domain & 190 & 202 & -12 & 0 & 0.94 \\
\hline$T c f 12$ & M2317 & Basic helix-loop-helix & 160 & 170 & -10 & 0 & 0.94 \\
\hline Gabpa & M4568 & ETS domain & 117 & 134 & -17 & 0 & 0.87 \\
\hline Etv2 & M5421 & ETS domain & 116 & 117 & -1 & 0 & 0.99 \\
\hline Zfp740 & M0429 & $\mathrm{C} 2 \mathrm{H} 2$-zinc finger & 98 & 120 & -22 & 0 & 0.82 \\
\hline Mesp1 & M5627 & Basic helix-loop-helix & 94 & 111 & -17 & 0 & 0.85 \\
\hline Tal1 & M6358 & Basic helix-loop-helix & 93 & 110 & -17 & 0 & 0.85 \\
\hline
\end{tabular}

nSykl, genes connected by given TF in Sykl network; nRapa, genes connected by given TF in Rapa network; nDiff, nSykl-nRapa; nOverlap, Genes overlapping between Sykl and Rapa; nRatio, nSykl/nRapa. ETV2 is among the TF with highest nDiff and is presented as bold entry.

nuclear translocation of transcription factors, including nuclear factor (erythroid-derived 2)-like 2 (Nrf2) and Ikaros (Uckun et al, 2012; Park et al, 2018). Therefore, we also examined the effect of Sykl and rapamycin treatment on ETV2 localization. Western blot analysis of ELT3-V cell fractions showed significantly increased nuclear ETV2 protein levels in Sykl-treated cells compared with DMSO-treated cells (Fig 2D and E), and importantly, treatment with rapamycin did not result in ETV2 nuclear accumulation. To further confirm the specificity of our findings, we silenced SYK in ELT3-V cells and similarly observed ETV2 nuclear accumulation (Fig S2A-G). These data collectively suggest that Syk inhibition, but not mTORC1 inhibition, drives ETV2 nuclear translocation.

\section{Etv2 silencing induces ER stress and leads to Tsc2-deficient cell death}

In addition to its role in endothelial and lymphatic lineage, ETV2's role as an important regulator of endothelial cell survival, cell cycle, and proliferation has been previously studied (Abedin et al, 2014; Singh et al, 2019). To assess the effect of ETV2 alteration on Tsc2deficient cells, we silenced EtV2 in ELT3-V cells (Fig 3A-C). Silencing Etv2 led to a significant increase in Annexin V-positive cells, indicating increased cell apoptosis/death with reduced ETV2 expression (Fig 3D and E). We then sought to determine whether the Etv2 silencing-mediated cell death is related to ER stress as Tsc2- deficient cells are known to be sensitive to ER stress (Ozcan et al, 2008; Kang et al, 2011). As shown in Fig 3, the expression of ER stress markers CHOP (Fig 3F and G) and phosphorylated ( $p$ )EIF-2 $\alpha$ (Fig 3F and $H$ ) were markedly elevated in Etv2-silenced ELT3-V cells, suggesting increased ER stress. Importantly, the ER stress response was associated with increased cell death as demonstrated by the increase in cleaved PARP (CPARP) expression (Fig 3F and I).

Contrarily, the cell death response was not observed when Etv2 was silenced in tuberin-reexpressing ELT3-T cells (Fig S3A-D). Moreover, silencing Etv2 induced cell death in Tsc2-deficient mouse embryonic fibroblasts, suggesting that the effect of Etv2 silencing is not cell type-specific (Fig S4A-D). These data strongly support the critical importance of ETV2, specifically in mTOR-hyperactivated cell survival.

To further confirm the ETV2-mediated induction of ER stress, we examined the role of ETV2 in the regulation of stress granule (SG) dynamics. We evaluated oxidant-induced SG formation in ELT3-V cells in which Etv2 was silenced by siRNA. ELT3-V cells transfected with Etv2 or Scr siRNA were exposed to arsenite $(0.5 \mathrm{mM})$ for $40 \mathrm{~min}$, and SG formation was quantified by immunofluorescence using the marker G3BP1 (Anderson \& Kedersha, 2002; Kosmas et al, 2021). ELT3-V cells transfected with EtV2 siRNA had a significantly increased number of SGs after arsenite treatment compared with cells transfected with Scr siRNA (1.5-fold increase; Fig 3J-K). These data suggest that ETV2 plays a role in SG assembly in response to oxidant stress. 


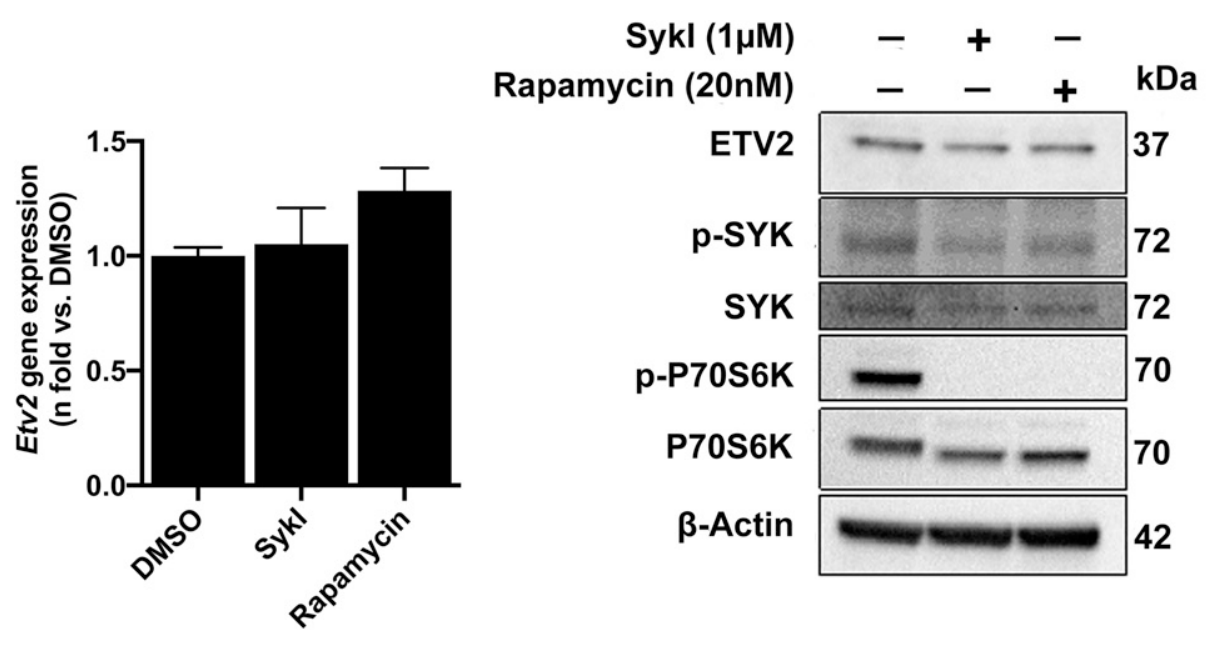

A

B

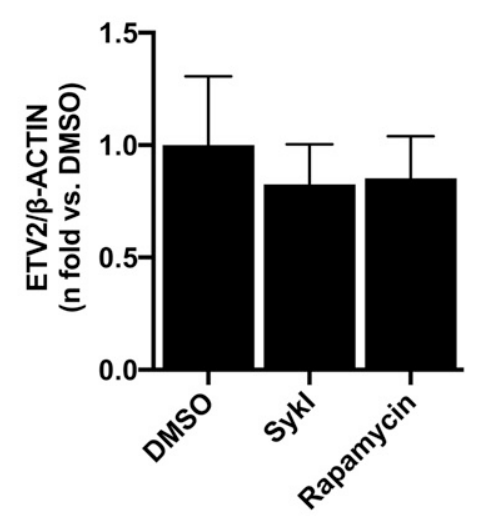

C
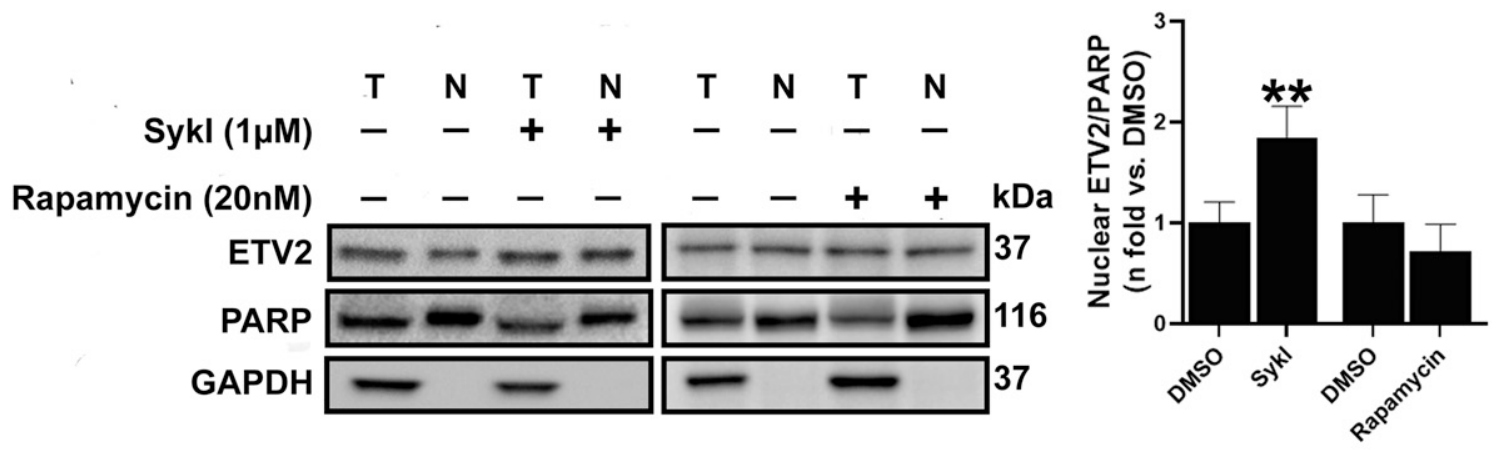

E

Figure 2. Effect of Sykl and rapamycin treatment on ETV2 expression and localization.

(A) Real-time qPCR analysis of rat Etv2 mRNA in Tsc2-deficient ELT3-V cells treated with Sykl or rapamycin for $24 \mathrm{~h}$ compared with DMSO-treated cells. Data represent mean \pm SEM of three independent experiments. (B, C) Immunoblot analysis of equal amount of rat ETV2 protein from ELT3-V cells treated with DMSO, Sykl, or rapamycin for $24 \mathrm{~h}$. Antibodies against rat ETV2, p-SYK, SYK, p-P70S6 kinase, and P70S6 kinase were used, and $\beta$-actin was used as a loading control. Band intensities of ETV2 were assessed, and ratios of ETV2/ $\beta$-actin were calculated for each treatment group. Results were expressed relative to DMSO. Data are means \pm SEM of at least three independent experiments (ETV2/ $\beta$-actin: $P>0.05$; $t$ test). (D, E) ELT3-V cells were treated with DMSO, Sykl, or rapamycin as in (B). Samples from equal fractions of total (T) and nuclear (N) protein lysates were separated by SDS-PAGE and transferred to polyvinylidene difluoride membranes, which were incubated with antibodies against ETV2, PARP (nuclear marker), and GAPDH (cytoplasmic marker). Band intensities of ETV2 were analyzed, and ratios of ETV2/PARP and ETV2/GAPDH were calculated for each group. Results were expressed relative to DMSO. Data are means \pm SEM of at least three independent experiments (ETV2/PARP: ${ }^{*} P<0.05,{ }^{* *} P<0.01 ; t$ test). Source data are available for this figure.

\section{Syk inhibition regulates Parpbp expression}

An examination of the PANDA analysis showed that ETV2 regulated 116 unique genes in the Sykl network and 117 unique genes in the rapamycin network. Parp1 binding protein (PARPBP) is an important component of DNA replication and damage response pathways and is differentially expressed in various cancers (Varisli, 2013; Feng et al, 2014; Uhlen et al, 2015). Based on our microarray data and PANDA analysis, we found that Parpbp was regulated by Etv2 uniquely under Sykl treatment and demonstrated the highest fold change between treatments compared with other ETV2-regulated genes within that network (Table 2).

RT-qPCR analysis showed that both Sykl and rapamycin treatments significantly reduced Parpbp expression compared with DMSO; however, the reduction was of significantly greater magnitude in the rapamycin treatment group than in Sykl (Fig S5A). We hypothesized that the relatively higher levels of Parpbp mRNA with Sykl treatment than with rapamycin treatment are due to ETV2 nuclear translocation. Accordingly, silencing of ETV2 significantly reduced overall Parpbp expression (Fig S5B), and abrogated the difference in the Parpbp expression level between the two treatment groups (Fig $4 \mathrm{~A}$ ), thus confirming the important role for ETV2 in Parpbp regulation in Tsc2-deficient cells.

Genome-wide analysis has revealed that ETV2 is an ETS factor with various transcriptional targets containing the consensus sequence of 5'-CCGGAA/T-3' and a core GGA/T motif (Wei et al, 2010; Lee et al, 2019). To examine whether ETV2 transcriptionally regulates Parpbp, Parpbp promoter fragments with one or no ETV2 consensus binding sequence were cloned into pGL3_Basic luciferase vector and transfected separately into ELT3-V cells with or without EtV2 

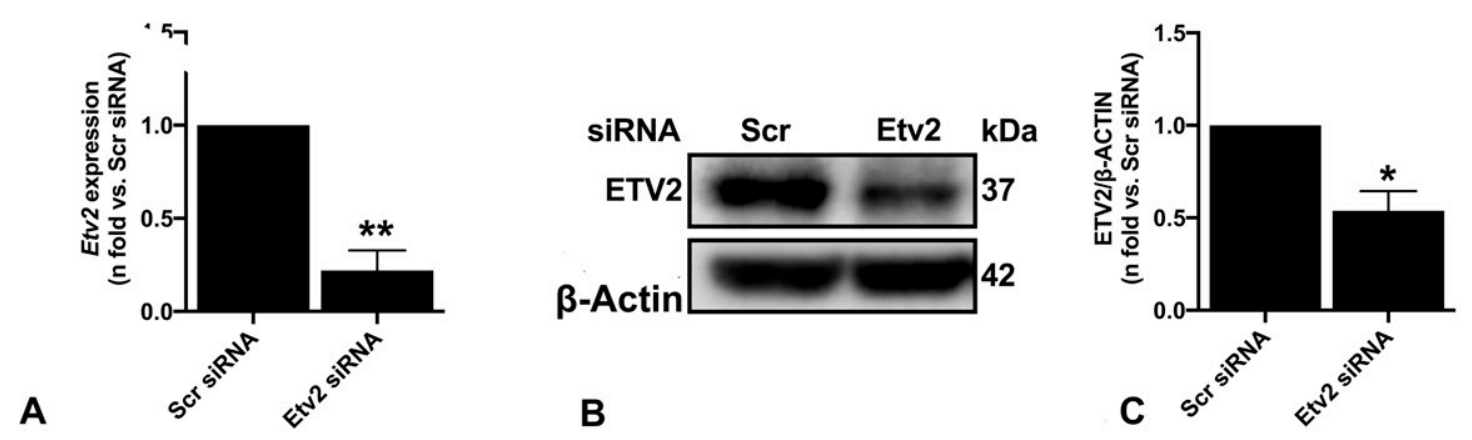

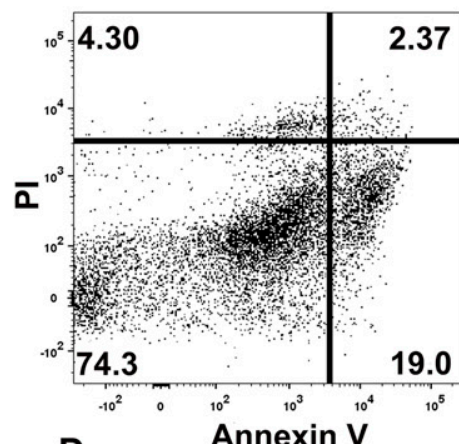

D

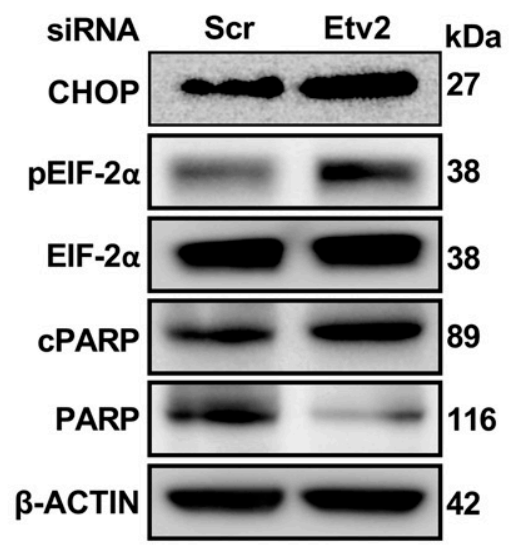

F

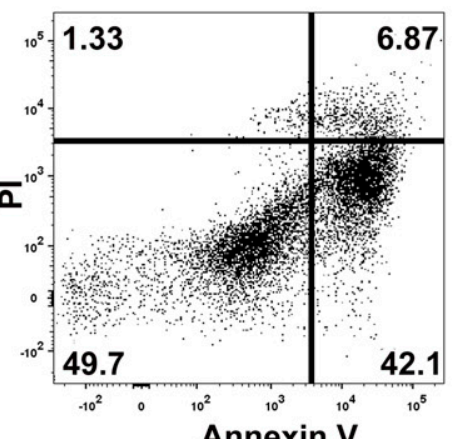

Annexin V

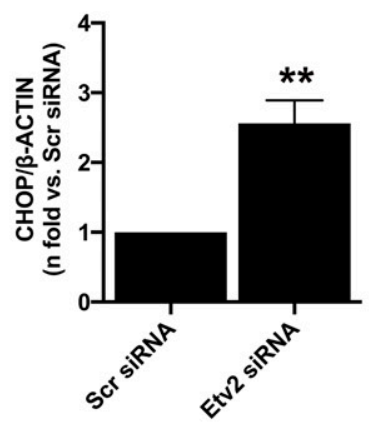

G
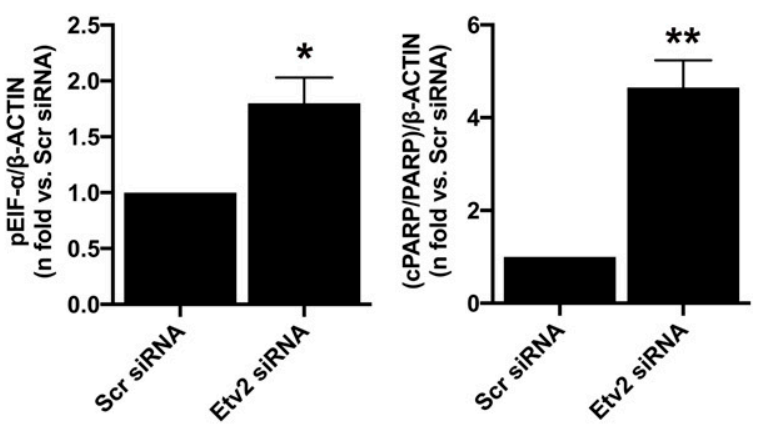

H

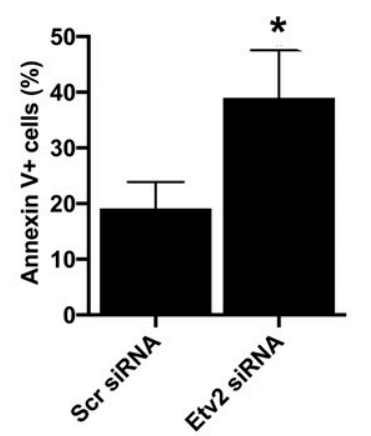

E 
silencing to determine promoter activity. The ELT3-V cells without Etv2 silencing transfected with pGL3_promoter-386 construct (i.e., the -311- to +75-bp putative promoter region) with one ETV2 consensus binding site displayed significantly higher luciferase activity than cells transfected with the pGL3_promoter-150 construct (i.e., the -75 - to +75-bp putative promoter region) with no ETV2 consensus binding site (Fig 4B). In addition, silencing of Etv2 significantly reduced the luciferase activity of the pGL3_promoter-386 construct but showed no effect in the pGL3_promoter-150 construct. These results demonstrated the role of ETV2 in Parpbp transcriptional regulation.

\section{Parpbp silencing leads to ER stress in Tsc2-deficient cells}

Parpbp silencing has been implicated in increased apoptosis and ER stress in myeloid leukemia cells (Nicolae et al, 2019). We hypothesized that ETV2-dependent ER stress and increased cell death in Tsc2-deficient cells are mediated by changes in PARPBP expression. To specifically investigate the role of PARPBP in the induction of ER stress and cell death in Tsc2-deficient cells, we silenced Parpbp in ELT3-V cells with Parpbp siRNA. Parpbp siRNA resulted in $\sim 80 \%$ and $50 \%$ reduction in Parpbp mRNA and PARPBP protein, respectively, compared with Scr siRNA (Fig 4C-E). Consequently, there was a significant increase in ER stress markers, including CHOP and pEIF-2 $\alpha$ (Fig 4F-H), and a significant increase in cell death marker, CPARP (Fig 4I). These observations support our hypothesis that the regulation of Parpbp expression by ETV2 contributes to ER stress and increased cell death in Tsc2-deficient cells following Etv2 silencing.

\section{Etv2 silencing leads to Tsc2-deficient cell death in vivo}

To determine the effect of Etv2 silencing on Tsc2-deficient cell survival in vivo, ELT3-V-luciferase cells were transiently transfected with Scr or Etv2 siRNA for $24 \mathrm{~h}$. Cells were intravenously injected into female SCID mice, and the level of bioluminescence was evaluated in the lungs 4-h postinjection. Similar levels of bioluminescence intensity were observed in mice injected with both Scr siRNA- and Etv2 siRNA-transfected cells (Fig 5A and B). By 24-h and 48-h postinjection, there was a decrease in bioluminescence in both groups; however, the bioluminescence intensities in the mice injected with Etv2-silenced cells were significantly lower than those in mice injected with Scr siRNA-transfected cells (Fig 5A and B). We then measured rat-specific DNA in cDNA samples obtained from lung tissue and peripheral blood using RT-qPCR. The levels of ratspecific DNA in both lung tissue and peripheral blood were significantly lower in the mice injected with Etv2-silenced cells than in the Scr control (Fig 5C and D). Consistent with our in vitro findings, these data suggest that Etv2 silencing leads to Tsc2-deficient cell death in vivo.

\section{ETV2 is expressed in human LAM samples}

Analysis of single-cell RNA-seq data from three LAM lung samples using Seurat identified seven different unique clusters of cells (Fig S6A), including alveolar type II (AT2) cells, conventional dendritic cells (CDC), endothelial cells, fibroblasts, macrophages, natural killer cells, and LAMCORE (Guo et al, 2020). A total of 121 LAMCORE cells defined as those which expressed ( $>0)$ LAM markers, PMEL, ACTA2, and FIGF (Guo et al, 2020) were identified (Fig 6A). One hundred LAMCORE cells were clustered distinctly, 13 were clustered within fibroblast population, and nine LAMCORE cells were distributed within other cell populations. We also examined ETV2 expression (>0) in LAMCORE cells and demonstrated that $96 \%$ of LAMCORE cells were positive for ETV2 expression (Fig 6A). Other cell types also expressed ETV2 however LAMCORE cells were among the highest expressing cells for in the LAM lung (Fig S6A).

Likewise, sorted cultured cells expressing CD44 and CD44V6 (known to have a loss of heterozygosity [ $\mathrm{LOH}]$ for TSC2) isolated from five different explanted lung samples (Samples 1-5, Fig 6B), and cells expressing CD235a isolated from eight blood samples from patients undergoing lung transplantation (Samples 6-13, Fig $6 \mathrm{~B}$ ) were analyzed by RT-PCR for ETV2 expression. Primers designed for PCR targeted the amplification of a 106-bp ETV2 mRNA sequence. The PCR product of the target region from each sample was visualized by electrophoresis on $2 \%$ agarose gels. All samples yielded a band at $106 \mathrm{bp}$, demonstrating the expression of ETV2 in all human LAM samples (primer only experimental control yielding no band, indicated by "Blank" is displayed in Fig S6B).

\section{Discussion}

We have previously shown that similar to mTORC1 inhibition, Syk inhibition leads to decreased proliferation of tuberin-deficient cells in vitro and in vivo (Cui et al, 2017). In this study, we used gene profiling and network-based approaches with PANDA analyses and identified ETV2 as a regulatory transcription factor uniquely altered under Syk inhibition and not under mTORC1 inhibitory conditions.

ETV2 is a well-known regulator of blood and endothelial cell lineages during development (Li \& Sidell, 2005; Garry, 2016). To date, the role of ETV2 in Tsc2-deficient tumors has not been investigated. Our data demonstrated that although Syk inhibition does not affect the expression of ETV2 at mRNA or protein levels, it prompted the translocation of ETV2 into Tsc2-deficient cell nuclei. Importantly,

were used to measure cell apoptosis induced by ETV2 silencing in ELT3-V cells. Representative flow cytometry plots for Scr and Etv2 siRNA are presented. The total percent of Annexin $V$ positive $(+)$ cells in each group was quantified from three independent experiments ( ${ }^{*} P<0.05 ; t$ test). ( $\left.\mathbf{F}, \mathbf{G}, \mathbf{H}, \mathbf{I}\right)$ Equal amounts of proteins were separated by electrophoresis and transferred to polyvinylidene difluoride membranes, which were reacted with antibodies against $\mathrm{CHOP}$, $\mathrm{pEIF-2} \alpha$, total EIF-2 $\alpha$, cleaved CPARP, and uncleaved PARP. $\beta$-Actin was used as a loading control. (G, H, I) Ratios of CHOP (G), pEIF-2 $\alpha /$ EIF-2 $\alpha$ (H), and cPARP/PARP (I) to $\beta$-actin were expressed as fold change to Scr siRNA. Data represent means $\pm \mathrm{SEM}$ of at least three independent experiments $\left({ }^{*} P<0.05,{ }^{* *} P<0.01 ; t\right.$ test). (J, $\left.\mathrm{K}\right)$ ELT3-V cells were treated with $0.5 \mathrm{mM} \mathrm{NaAsO} \mathrm{O}_{2}$ (arsenite) for $40 \mathrm{~min}$. Cells were stained for G3BP1 (green) to detect stress granules, and DAPI (blue) was used to visualize the nuclei. A total of 221 cells per siRNA group were imaged and quantified with CellProfiler. Scale bar, $20 \mu \mathrm{m}$, ${ }^{\star * * \star} P<0.0001$.

Source data are available for this figure. 
Table 2. List of top 10 genes identified with PANDA analysis regulated by Etv2 included in the Sykl-specific network.

\begin{tabular}{lllll} 
Gene symbol & Gene name & Fold change & P-value & False discovery rate q-value \\
\hline Parpbp & PARP1 binding protein & -1.38 & 0.00 & 0.05 \\
\hline Fam111a & Family with sequence similarity 111, member A & -1.22 & 0.00 & 0.03 \\
\hline Ppp1r16b & Protein phosphatase 1, regulatory subunit 16B & 1.22 & 0.03 & 0.38 \\
\hline Mtus1 & Microtubule-associated scaffold protein 1 & 1.16 & 0.01 & 0.29 \\
\hline Adarb2 & Adenosine deaminase, RNA-specific, B2 & 1.16 & 0.01 & 0.25 \\
\hline Gldn & Gliomedin & -1.14 & 0.02 & 0.35 \\
\hline Rnf219 & Ring finger protein 219 & -1.14 & 0.03 & 0.37 \\
\hline Il20rb & Interleukin 20 receptor subunit beta & -1.13 & 0.01 & 0.23 \\
\hline Vamp1 & Vesicle-associated membrane protein 1 & 1.11 & 0.04 & 0.41 \\
\hline Gprc6a & G protein-coupled receptor, class C, group 6, member A & -1.10 & 0.03 & 0.40 \\
\hline
\end{tabular}

rapamycin treatment does not affect the expression or nuclear localization of ETV2, suggesting that ETV2 may drive a potential mTORC1-independent transcriptional pathway in Tsc2-deficient cells. The presence of cAMP response element (CRE) sequences and therefore the regulation of ETV2 by PKA signaling have been shown previously (Yamamizu et al, 2012). Likewise, the interaction between SYK and PKA has also been described (Yu et al, 2013). Therefore, the nuclear translocation of ETV2 during Sykl treatment could potentially be driven in a PKA-dependent manner. Further elucidation of this pathway will require additional investigation.

A known target of ETV2, acting as a transcription factor, are genes involved in the regulatory networks for hematopoietic and endothelial lineages, including Flk1 (Kim et al, 2019). We used PANDA network analysis and identified multiple genes that could be potential transcriptional targets of ETV2 following Syk inhibition in Tsc2-deficient cells. The most differentially regulated gene was Parpbp. Interaction between ETV1, EWS-ERG fusion genes, and EWSFLI1 fusion genes, members of ETS family transcription factors, and PARP1, a PARPBP-interacting partner has been demonstrated in Ewing sarcoma and prostate cancers (Feng et al, 2014). Our study is the first to demonstrate that ETV2 transcriptionally regulates Parpbp. Both Sykl and rapamycin result in mTORC1 blockade (Cui et al, 2017). Our data showed that treatment with both SykI and rapamycin resulted in decreased Parpbp levels, which we attribute to mTORC1 blockade. The rebound increase in Parpbp levels with Syk inhibition is likely due to ETV2 nuclear translocation, which is seen only with Syk inhibition and not mTORC1 inhibition.

More importantly, to investigate the functional role of ETV2 in Tsc2-deficient cells, we silenced Etv2 and demonstrated that silencing of EtV2 induced ER stress, leading to increased cell death both in vitro and in vivo. Our finding of ER stress-mediated Tsc2deficient cell death extends prior knowledge on the contribution of ETV2 to cell survival and apoptosis (Abedin et al, 2014; Singh et al, 2019) as well as the susceptibility of Tsc2-deficient cells to ER stress (Ozcan et al, 2008; Kang et al, 2011). It was recently shown that TSC2 physically interacts with the SG protein G3BP1 and that SGs are increased in Tsc2-deficient cells (Kosmas et al, 2021). SGs may represent a mechanism to temporarily sequester transcripts during transient stress, including oxidant stress. Our data showed that silencing Etv2 increased SGs in Tsc2-deficient cells in the setting of short-term arsenite-induced oxidant stress, suggesting that multiple mechanisms impact SGs in TSC. Furthermore, we also demonstrated that Parpbp silencing, similar to silencing of Etv2, resulted in increased ER stress and increased cell death in Tsc2-deficient cells. This suggested that ETV2 induces ER stress and increases cell death in Tsc2-deficient cells via Parpbp regulation (Fig 7). We also showed that MEFs deficient in tuberin are also sensitive to ETV2 depletion, resulting in cell death. Importantly, we also showed that cells expressing TSC2 are not susceptible to ETV2-mediated cell death.

ELT3 cells share some important common features with LAM cells including tuberin deficiency, activation of MTOR, and perhaps a common uterine origin (Guo et al, 2020). We elected to use ELT3 cells in an in vivo model of Tsc2-deficient cell colonization to the lung. At 4-h postinjection, there was no difference in bioluminescence intensity over the lungs between cells where Etv2 was silenced and controls, suggesting that an identical number of cells successfully colonized the lungs. The decrease in bioluminescence over the subsequent time points in cells where Etv2 was silenced strongly supports our hypothesis that ETV2 is critical for tuberin-deficient cell survival in vivo. Finally, we validated ETV2 expression in human LAM cells in three different ways. First, we identified LAM cells in the recently published single-cell RNAseq data and found that these cells overwhelmingly expressed ETV2. Second, in a mixed cell population isolated from LAM lungs, we found that cells with $\mathrm{LOH}$ for TSC2 expressed ETV2. Finally, LAM cells isolated from peripheral blood from female patients with LAM also expressed ETV2.

Taken together, the combination of the in vitro, in vivo, and human data strongly supports our hypothesis of a critical role for ETV2 in Tsc2-deficient cell survival (Fig 7A and B). Therefore, identifying molecules that target ETV2 expression or prevent its nuclear trafficking could potentially be of important therapeutic benefit in LAM and other tumors driven by mTORC1 activation.

\section{Materials and Methods}

\section{Cell culture}

Tsc2-deficient Eker rat uterine leiomyoma (ELT3-V) cells were cultured using DMEM containing 10\% fetal bovine serum and 

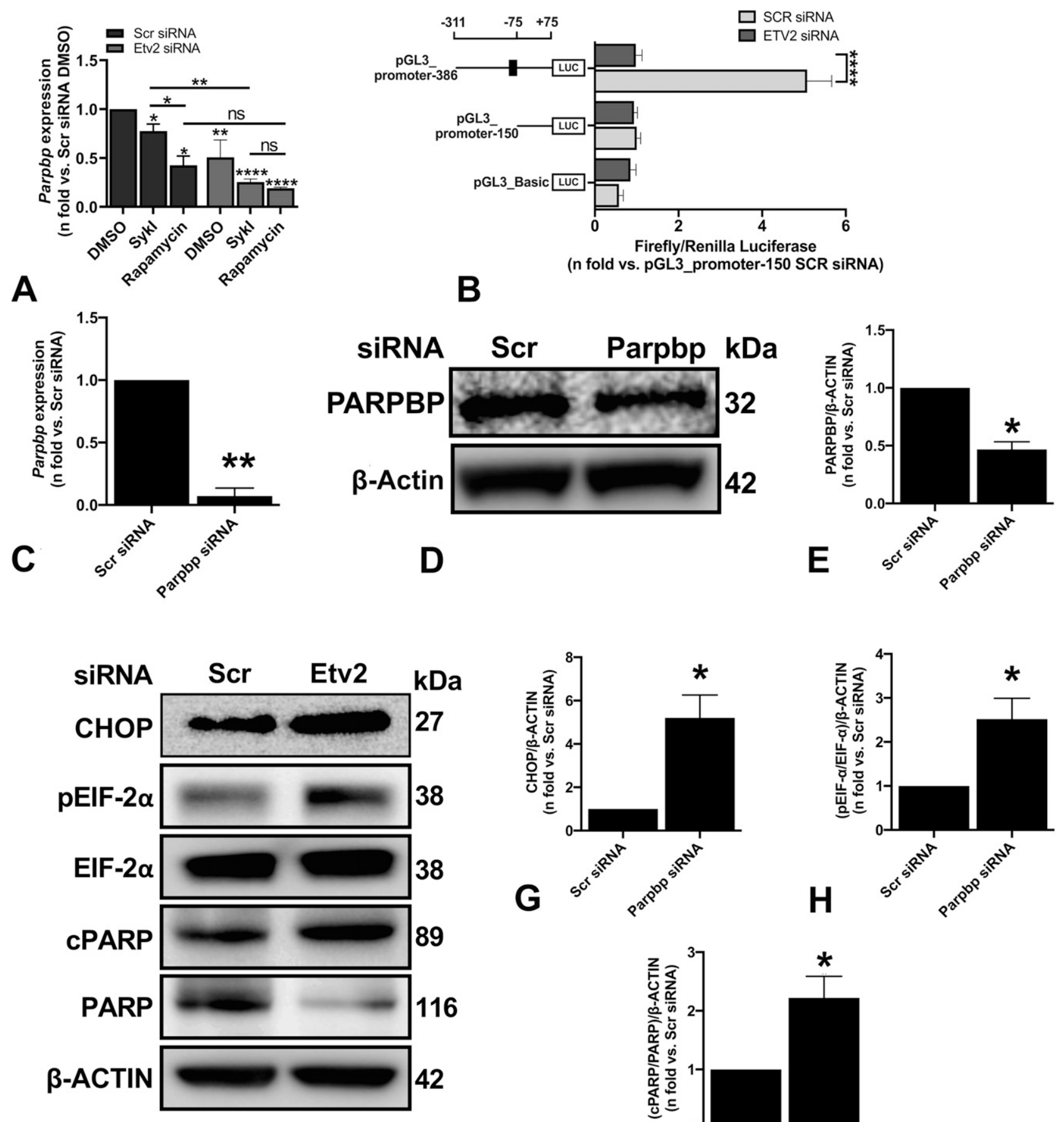

$\mathbf{F}$
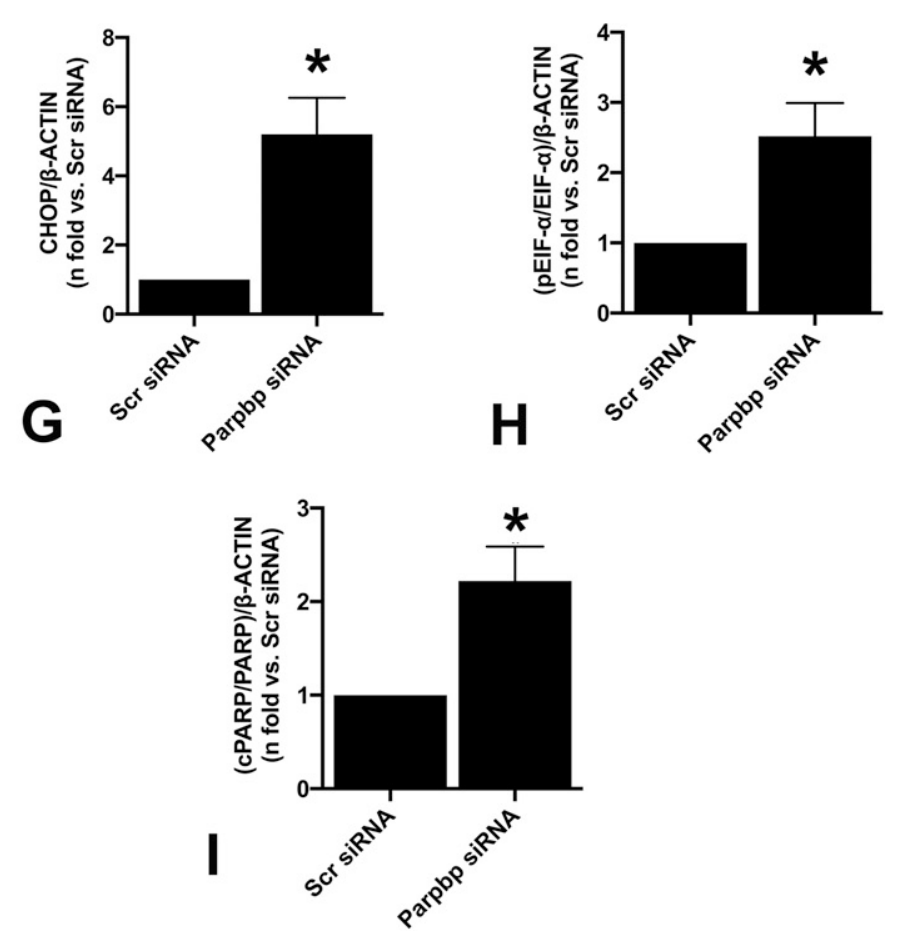

Figure 4. Etv2 silencing-induced changes in PARPBP lead to ER stress and cell death in Tsc2-deficient cells.

(A) ELT3-V cells were transfected with Scr siRNA and Etv2 siRNA for $24 \mathrm{~h}$ and treated with DMSO, Sykl, or rapamycin for an additional $24 \mathrm{~h}$. RT-qPCR was carried out on an equal amount of total RNA $(1 \mu \mathrm{g})$ converted to cDNA to analyze the transcript levels of Parpbp gene. The histogram represents fold change in mRNA expression relative to Scr siRNA with DMSO treatment. Data are means \pm SEM of at least three independent experiments $\left({ }^{*} P<0.05,{ }^{* *} P<0.01,{ }^{* *} P<0.001 ; t\right.$ test). (B) ELT3-V cells were transfected with Scr siRNA and Etv2 siRNA for $24 \mathrm{~h}$, followed by transfection with internal control, pGL3_Renilla plasmid, and pGL3_Basic vector or Parpbp promoter constructs for an additional $24 \mathrm{~h}$. Firefly luciferase and Renilla luciferase activities of negative control, pGL3_Basic vector and Parpbp promoter constructs with and without ETV2-binding site, pGL3_promoter-386, and pGL3_promoter-150 were measured. Each set of luciferase data is normalized to internal Renilla control. Data are presented relative to the pGL3_promoter-150 Scr siRNA sample. Data are means \pm SEM of at least three independent experiments ( ${ }^{\star * \star *} P<0.0001 ; t$ test). (C) ELT3-V was transfected with either Scr or Parpbp siRNA for $48 \mathrm{~h}$. Real-time qPCR analysis of rat Parpbp mRNA was performed. The histogram represents fold change in mRNA expression compared with the Scr 
incubated at $37^{\circ} \mathrm{C}$ in a humidified $5 \% \mathrm{CO}_{2}$ atmosphere. For each experiment, cells were serum-starved overnight (16 h) prior to treatment with the vehicle control, DMSO (Sigma-Aldrich), Syk inhibitor (Sykl) R406 (1 $\mu \mathrm{M}$; Selleckchem), or rapamycin (20 nM; LC Laboratories).

\section{GeneChip hybridization and differential expression analyses}

ELT3-V cells were treated with DMSO, rapamycin, or Sykl for $24 \mathrm{~h}(\mathrm{n}=4$ biological replicates per group). RNA was extracted using the RNeasy Mini Kit (QIAGEN). All subsequent sample preparation and GeneChip (Rat Gene 2.0 ST arrays) processing were performed at the Boston University Microarray and Sequencing Resource Core facility using an input of $1 \mu \mathrm{g}$ of total RNA from each sample. Analysis was performed using the Bioconductor software suite (version 2.12) (Gentleman et al, 2004). Chip definition file (Dai et al, 2005) was processed using a robust multi-array average (RMA) algorithm (Irizarry et al, 2003) available in the affy package (version 1.36.1) (Gautier et al, 2004). The $\log _{2}$ scale data from RMA were used in statistical testing.

Differential expression analysis was performed, and the BenjaminiHochberg false discovery rate (Benjamini \& Hochberg, 1995) was implemented for both ANOVAs and $t$ tests to generate corrected $P$-values ( $q$ values). A filtered gene list was generated for expression changes of greater than 2.0-fold and one-way ANOVA false discovery rate $q<0.01$ and, furthermore, divided into four distinct clusters based on the expression pattern in the three conditions to generate a heatmap. Principal component analysis was performed by normalizing gene expression values across all samples to a mean of 0 and an SD of 1.0 with the prcomp R function. The three treatment conditions were separated with the first principal component (PC1), and replicates in each condition were separated with the second PC2.

\section{Pathway analyses}

GO term enrichment analysis of clustered gene groups was performed using DAVID (Huang et al, 2009a, 2009b) with default settings. HomoloGene (version 68) (NCBI Resource Coordinators, 2013) was used to identify human homologs of the rat genes in the array. The $\mathrm{R}$ environment (version 3.4.3) was used for all microarray analyses. Kyoto Encyclopedia of Genes and Genomes (KEGG) pathway analysis was used to perform subsequent bioinformatics analysis of all the genes identified in microarray, irrespective of the clusters. Both GO term and KEGG analysis pathways were selected with a $P$-value $<0.05$ and a gene count $>2$.

\section{Transcription factor and target network construction}

Passing attributes between networks for data assimilation (PANDA) analysis was used to construct gene regulatory networks for each of three treatment drugs, Syk, Rapa, and DMSO, using all genes in the microarray dataset, as previously described (Glass et al, 2013). An initial map of transcription factors to genes was created by scanning the rn6 genome for 620 Cis-BP Rattus norvegicus motifs provided with the MEME suite (Bailey et al, 2009) using the Finding Motif Occurrences (FIMO) program (Grant et al, 2011). Statistically significant $\left(p<1 \times 10^{-4}\right)$ hits within the promoter region, defined as a the $[-750,+250]-b p$ region around the transcriptional start site of RefSeq annotated genes, were retained. The initial mapping included 2,245,143 edges (17,177 genes, 616 TF). There were 14,890 genes and 616 TF common to both expression data and the motif mapping. Potential inferred regulatory relationships were determined by using PANDA to integrate the motif mapping and gene expression data and assign a weight (z-score) to each edge that connects a TF to its target gene. Top 10,000 (TF, gene) pairs with the largest absolute differences of edge weights in three different pairwise comparisons were generated: Pair 1. E(SykI)-E(DMSO), Pair 2. E(rapamycin)-E(DMSO), and Pair 3. E(rapamycin)-E(SykI). In addition, edge weight differences for Pair 3 were plotted as scatter plots for visualization using Cytoscape. Transcription factors involved in the top edges for each pairwise comparison were identified from the network of differential regulation.

\section{Real-time (RT)-qPCR}

$1 \mu \mathrm{g}$ of total RNA extracted from ELT3-V cells using the RNeasy Mini Kit (QIAGEN) was reverse-transcribed into cDNA using amfiRivert CDNA Synthesis Master Mix (GenDEPOT). Quantitative real-time polymerase chain reaction (qPCR) was performed using iTaq Universal SYBR Green qPCR Master Mix (Bio-Rad), according to the manufacturer's protocol. Table S1 provides the primer sequences used in qPCR analyses.

\section{Cellular fractionation}

Treated ELT3-V cells were washed with cold PBS and collected by scraping. Cellular fractionation was performed using a Cellytic NuCLEAR Extraction Kit (Sigma-Aldrich), according to the manufacturer's instructions. A portion of cell pellets was used for total protein isolation using RIPA buffer (Thermo Fisher Scientific). Both nuclear and total protein isolation buffers were supplemented with protease and phosphatase inhibitors (Invitrogen). Equal fractions of lysates for both nuclear and cytoplasmic fractions were subjected to immunoblotting.

\section{Immunoblot}

Equal amounts or fractions of indicated protein lysates were loaded onto NuPage $4-12 \%$ Bis-Tris Protein Gels (Invitrogen) and then

control. Data are means \pm SEM of at least three independent experiments (** $P<0.01 ; t$ test). (D, E) Equal amounts of protein from whole-cell lysates of ELT3-V transfected with either Scr or Parpbp siRNA for $48 \mathrm{~h}$ were analyzed by Western blotting with antibodies against Parpbp and $\beta$-actin. The ratio of Parpbp to $\beta$-Actin density was expressed as the fold-change relative to Scr siRNA. Data are means \pm SEM of at least three independent experiments ( ${ }^{\star} P<0.05 ; t$ test). ( $\left.\mathbf{F}, \mathbf{G}, \mathbf{H}, \mathbf{I}\right)$ Equal amounts of proteins were separated by electrophoresis and transferred to a polyvinylidene difluoride membrane which were reacted with antibodies against $\mathrm{CHOP}$, $\mathrm{pEIF-2} \alpha$, total EIF-2 $\alpha$, CPARP, and uncleaved PARP. $\beta$-Actin was used as a loading control. (G, H, I) Ratios of CHOP (G), pEIF-2 $\alpha /$ EIF-2 $\alpha$ (H), and cPARP/PARP (I) to $\beta$-actin density was expressed as fold change to Scr siRNA. Data represent means \pm SEM of at least three independent experiments $\left({ }^{\star} P<0.05, t\right.$ test).

Source data are available for this figure. 

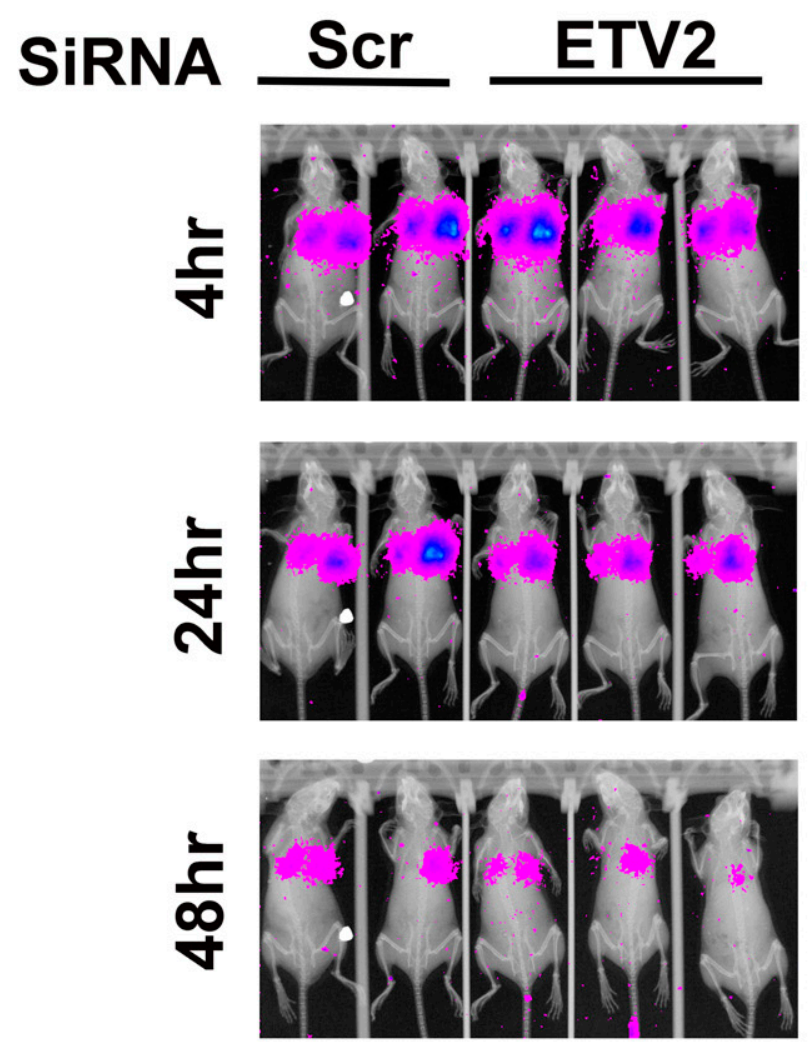

A
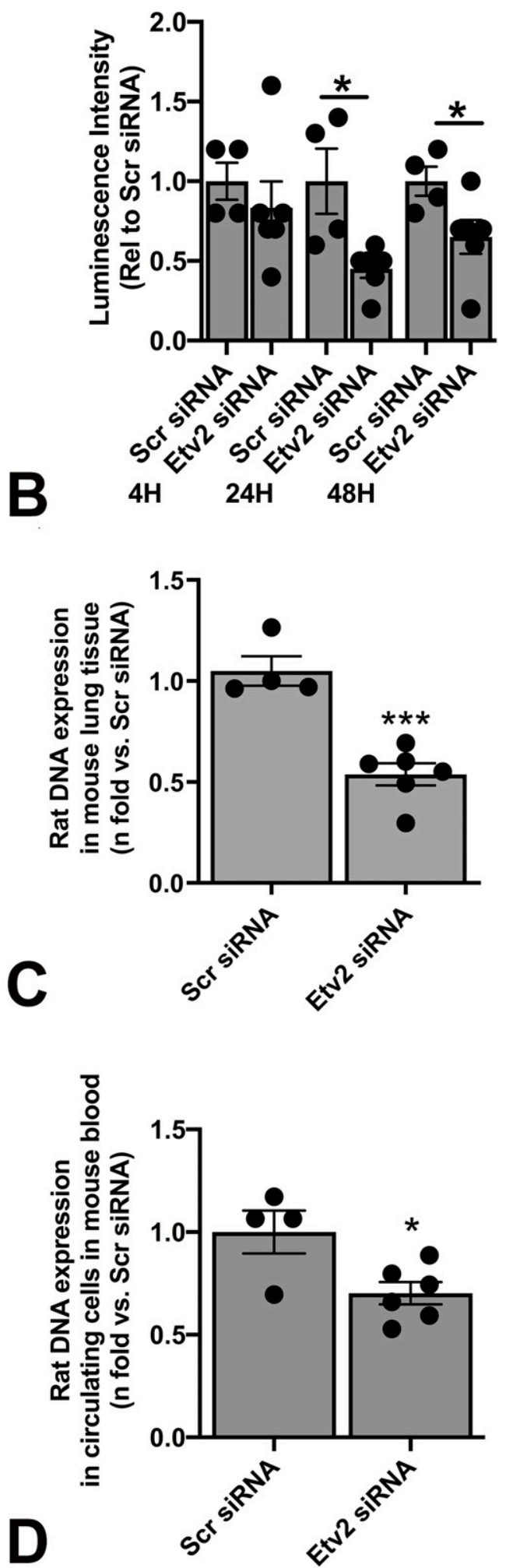

Figure 5. Silencing Etv2 leads to Tsc2-deficient cell death in vivo.

(A) ELT3-V-luciferase cells were transfected with Scr and Etv2 siRNA for $24 \mathrm{~h} .1 \times 10^{6}$ cells were injected in C.B17 Scid mice (Scr siRNA $n=4$; Etv2 siRNA $\mathrm{n}=6$ ) via lateral tail vein injection. Bioluminescence was measured using Bruker In-Vivo Xtreme to show lung colonization of ELT3-V-luciferase cells at 4, 24 , and $48 \mathrm{~h}$. Representative images are presented. (B) Net luminescence intensity was accessed for each mouse at each time point. Histogram reveals a decrease in luminescence intensity in the Etv2 siRNA group relative to the $\mathrm{Scr}$ siRNA group at each time point. ( ${ }^{*} P<0.05$; $t$ test). (C) 48 -h postinjection, aortic blood was collected from each mouse. Levels of circulating cells from Scr siRNA $(n=4)$ and Etv2 siRNA $(n=6)$ mice were measured by RT-qPCR using rat-specific primers. The histogram represents fold change in rat DNA expression 
subsequently immunoblotted with the primary antibodies listed in Table S2. Each protein of interest was then detected with HRPconjugated goat anti-rabbit or anti-mouse IgG antibody (1:2,000; Invitrogen) and visualized using Supersignal West Pico PLUS Chemiluminescent Substrate or SuperSignal West Femto Maximum Sensitivity Substrate (Thermo Fisher Scientific).

\section{RNA interference}

Predesigned MISSION siRNA targeting rat EtV2 and rat Parpbp, as well as MISSION siRNA Universal Negative control, were purchased from Sigma-Aldrich. ELT3-V cells were transfected with Etv2 siRNA (10 $\mu \mathrm{M})$, Parpbp siRNA ( $5 \mu \mathrm{M})$, or scrambled (Scr) siRNA using Lipofectamine RNAiMAX reagent (Thermo Fisher Scientific) and OPTI-MEM (Thermo Fisher Scientific). Sequences for siRNA used are listed in Table S3.

\section{Arsenite-induced SG formation}

ELT3-V cells were plated on four-well chamber tissue culture slides (Corning) and transfected with Scr or Etv2 siRNA. 48-h posttransfection, cells were treated with $0.5 \mathrm{mM}$ sodium arsenite $\left(\mathrm{NaAsO}_{2}\right)$ (MilliporeSigma) for $40 \mathrm{~min}$. The cells were then fixed with $4 \%$ paraformaldehyde and processed for immunofluorescence analysis using GAP SH3 Binding Protein 1 (G3BP1) antibody (green; Abcam) and fluorophore-conjugated secondary antibody. Nuclei were visualized with 4',6-diamidino-2-phenylindole (DAPI, blue; Sigma-Aldrich) staining. Images were captured with a FluoView FV10i Olympus Laser Point Scanning Confocal Microscope using a 60× objective (Olympus).

\section{Luciferase reporter assay}

The Parpbp promoter fragments with (386 bp) or without (150 bp) one putative ETV2 binding site (Wei et al, 2010) were cloned into pGL3_Basic vector (Cat. no. E1751; Promega). Primers forward: taagcagagctcgtcggagggcgagcgaggcg and reverse: tgcttaaagctttaccacgatgccgctggagg were used to clone -311 to +75 bp and primers forward: taagcagagctcggcgcggaacaagcgtagtagtcag and reverse: tgcttaaagcttttaccacgatgccgctggagg were used to clone -75 to +75 bp promoter fragments. Reverse transfection was carried out in a total of 15,000 ELT3-V cells transfected with Scr and Etv2 siRNA for $24 \mathrm{~h}$ using X-tremeGENE HP DNA transfection reagent (Sigma-Aldrich). Cells were transfected with 10 ng of pRL_CMV vector (internal control, Cat. no. E2261; Promega) and 140 ng of promoter constructs. Luciferase activity was tested $24 \mathrm{~h}$ after plasmid transfections using the Dual-Glo Luciferase Assay System kit (Cat. no. E2920; Promega) as per the manufacturer's protocol. Each transfection was carried out in triplicates in four independent experiments. Biotek Synergy HT microplate reader (BioTek) with Biotek Gen5.1.1 microplate data collection software was used for luciferase luminescence detection.

\section{Animal studies}

All animal experimental procedures were performed according to protocols approved by the Institutional Animal Care and Use Committee at Brigham and Women's Hospital. ELT3-V cells stably transduced with pCMV-luciferase (ELT3-V-luciferase) were transfected with Scr siRNA and Etv2 siRNA. 24-h post-transfection, $1 \times 10^{6}$ cells were injected into female immunodeficient C.B17 SCID mice (Taconic) via lateral tail vein injections. Before imaging, mice were injected with the RediJect D-luciferin bioluminescent substrate (Cat. no. 770504; Perkin-Elmer). Bioluminescent signals were recorded at 4, 24, and $48 \mathrm{~h}$ using an In-Vivo Xtreme imaging system (Bruker). Net luminescence intensity in the chest area was assessed using Bruker molecular imaging (MI) software (v7). Murine blood was collected at the end of the experiment by aortic puncture, and red blood cells were lysed using an ammonium-chloride-potassium (ACK) lysing buffer (Quality Biological). In addition, murine lung tissues were harvested, minced, and enzymatically digested (300 units/ml Collagenase 4; Worthington Dorchester). DNA was extracted from blood and mouse lung tissues using a Blood and Tissue DNA kit (QIAGEN). Rat and mouse DNA were quantified by RT-qPCR using rat- and mouse-specific primers included in Table S1 (Walker et al, 2004; Yu et al, 2009).

\section{Analysis of available single-cell RNA-Seq data}

SCRNA seq-data for four LAM samples were downloaded from Gene Expression Omnibus (GSE135851) (Guo et al, 2020). Previous analysis of LAM sample 2 did not detect LAMCORE positive cells and therefore was excluded from our analysis. The data from LAM samples 1, 3, and 4 were analyzed using Seurat v3 (Butler et al, 2018). For the three LAM samples, genes expressed in at least two cells were selected. Furthermore, for LAM samples 1 and 3 specifically, cells with at least 500 expressed genes (nFeature_RNA > 500) with less than $10 \%$ of genes mapping to the mitochondria (percent.mt < 10) were selected. For LAM sample 4 , nuclei with at least 300 expressed genes and with less than $10 \%$ of genes mapping to the mitochondria were included for analysis. The three Seurat objects were merged into a single object and preprocessed using the outline previously described (Butler et al, 2018). The samples were then integrated using the $\mathrm{R}$ package Harmony (Korsunsky et al, 2019), followed by imputation of the data using the R package Alra (Linderman et al, 2022). Clusters were then generated using Seurat and labeled with the R package ClustifyR (Fu et al, 2020) using previously published data as a reference matrix (Habermann et al, 2020). LAMCORE cells were identified based on positive $(>0)$ expression of LAM markers PMEL, FIGF, ACTA2, and VEGF-D (Guo et al, 2020); 100 of these cells clustered together in the LAMCORE cluster. ETV2-positive cells within the LAMCORE cells were identified by positive (0>) expression of ETV2.

relative to Scr siRNA. (D) Rat DNA in the mouse lungs harvested 48-h postinjection from Scr siRNA ( $n=4)$ and Etv2 siRNA ( $n=6)$ mice were measured by RT-qPCR using rat-specific primers. The histogram represents fold change in rat DNA expression relative to Scr siRNA. 


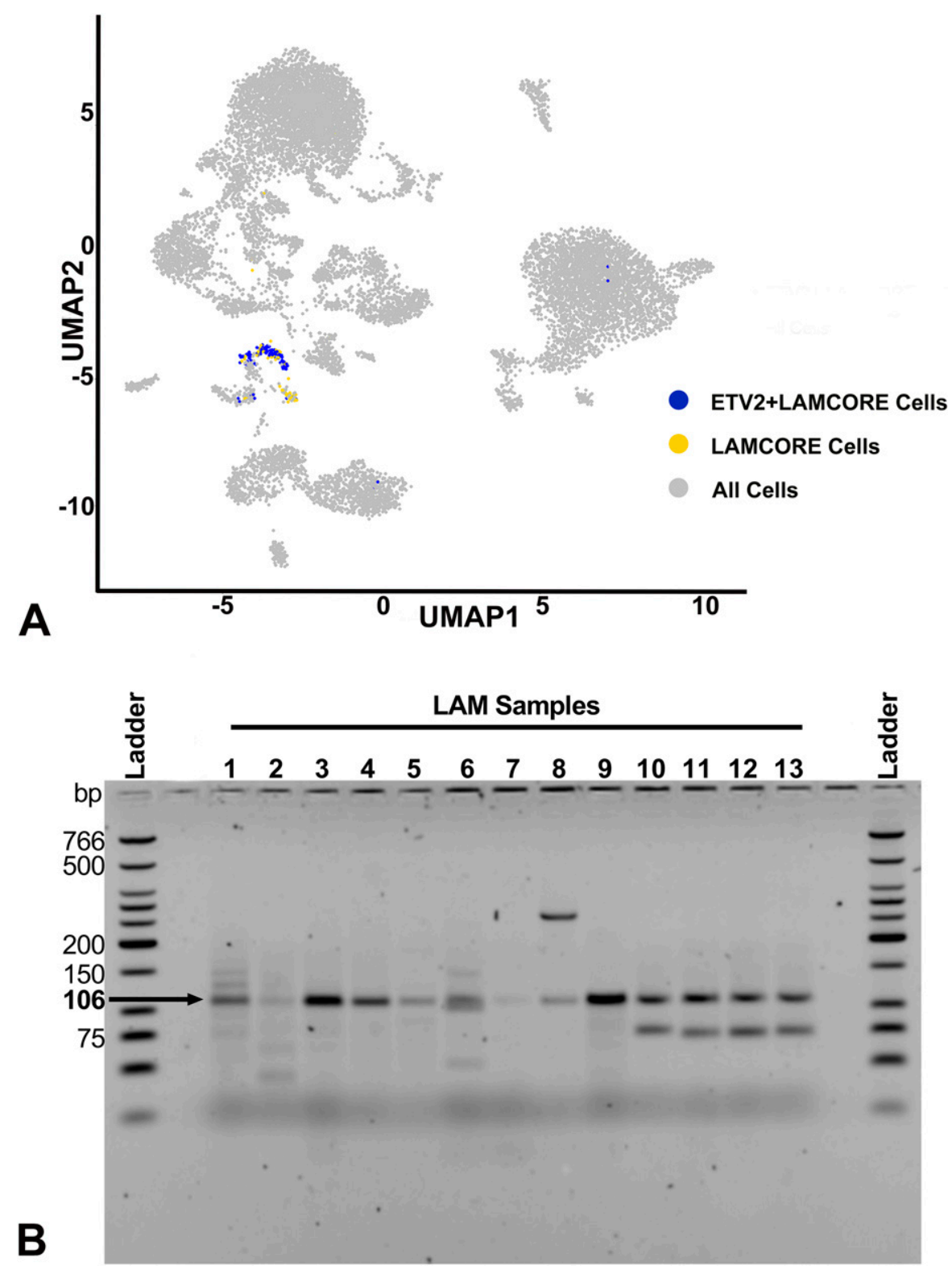

Figure 6. ETV2 expression in human LAM samples. (A) Single cells RNAseq analysis of LAM samples detecting ETV2 expressing cells in the LAMCORE population. Feature plot indicates 15 different clusters of cells (gray) and a distinct cluster of LAMCORE cells (yellow), in the LAM samples. ETV2-expressing cells within the LAMCORE cells are indicated in blue. (B) Agarose gel electrophoresis analysis of ETV2 expression in cultured LAM cells isolated from explanted lungs of patients undergoing lung transplantation (Samples 1-5) and circulating cells from human blood (Samples 6-13). Amplicons of 106 bp were obtained in all samples.

Source data are available for this figure.

\section{ETV2+LAMCORE Cells}

All Cells

\section{Isolation of cells with LOH for TSC2}

Cells isolated from explanted lungs of patients undergoing lung transplantation were cultured in mesenchymal stem cell media (MSCGM) as described previously (Pacheco-Rodriguez et al, 2007). Cultured cells $(\sim 1 \times$ $10^{6}$ ) were reacted with $20 \mu \mathrm{l}$ antibodies of each anti-CD44V6-FITC and antiCD44-PE (Table S2), following trypsinization. Cells underwent a 30-min incubation at room temperature. Then, $3 \mathrm{ml}$ of PBS was added, and the tubes were centrifuged for $10 \mathrm{~min}$ at $\sim 250 \mathrm{~g}$. The cell pellet was then suspended in $500 \mu \mathrm{l}$ PBS. Cell mixtures were placed on ice until sorted using a BD FACSaria 2 (Becton Dickinson). Four subpopulations of cells were obtained in PBS and RNA later (Sigma-Aldrich). We previously showed that cells sorted expressing CD44 and CD44v6 were more likely to have LOH for TSC2. To identify circulating LAM cells, we took $60 \mathrm{ml}$ of heparinized fresh blood to isolate cells, following a density gradient. For that purpose, we used CD235a-PE and CD45-FITC antibodies (Table S2). Cells with TSC2 LOH are most likely to be found in cells expressing CD235a. TSC2 LOH was determined using five microsatellite markers (D16S521, D16S3024, D16S3395, Kg8, and D16S291) on chromosome 16, as previously described (Steagall et al, 2013).

Total RNA was isolated from cells in RNALater, cDNA synthesized, and qPCR performed, according to the manufacturer's protocol. Primer sequences used in QPCR analyses were provided in Table S1. PCR products were subjected to agarose gel (2\%) electrophoresis to visualize the amplified ETV2 qPCR products.

\section{Statistical analysis}

For all experiments, at least three independent experiments were conducted, and data are presented as mean \pm SEM. Statistical 


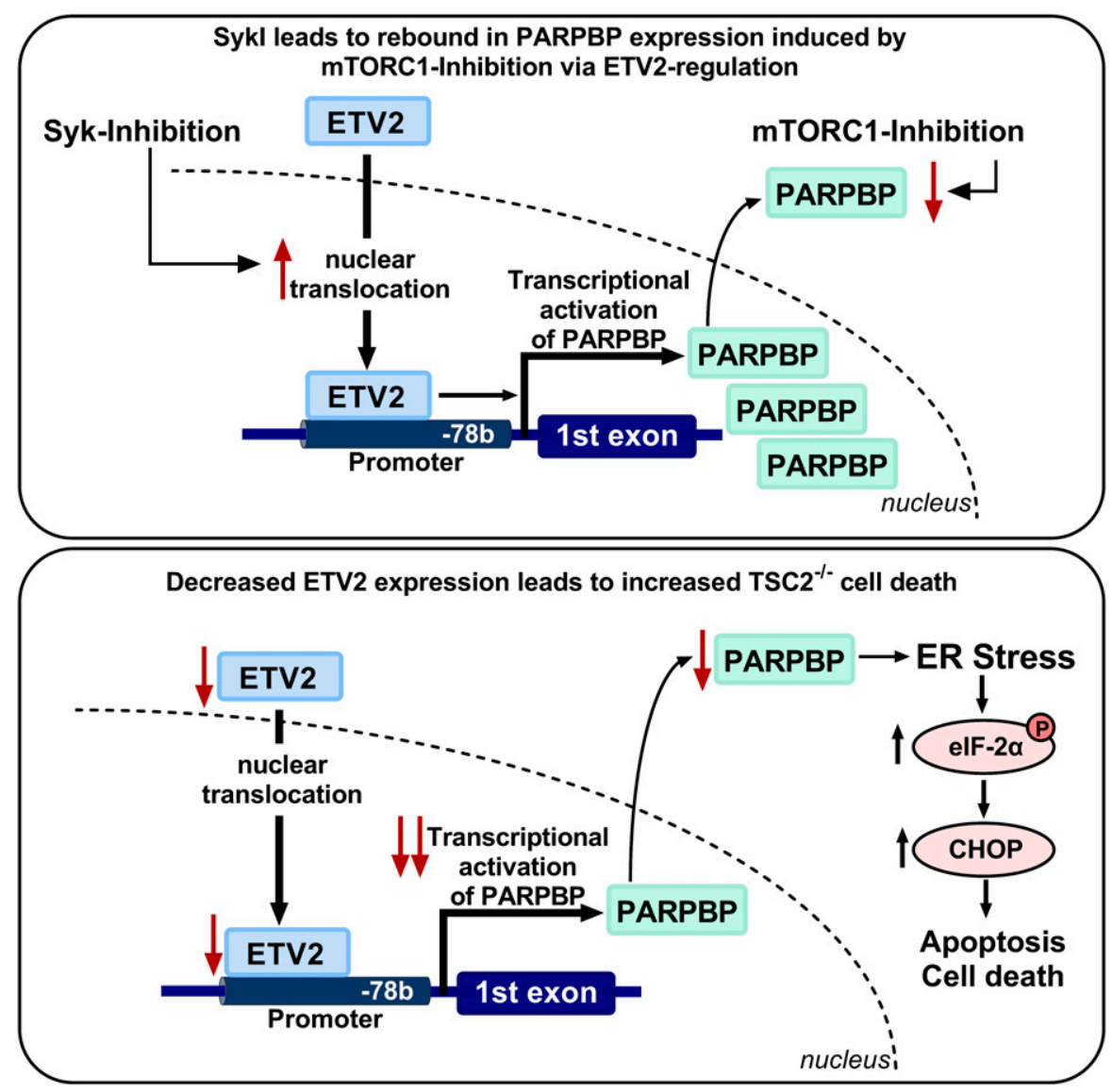

Figure 7. Schematic representation of ETV2 nuclear translocation and PARPBP regulation.

(Top panel) In Tsc2-deficient cells, Syk inhibition (Sykl) drives ETV2 to the nucleus, where it binds to its consensus sequence in the Parpbp promoter at position -78 base pair to transcriptionally activation of Parpbp gene expression. (Bottom panel) In Tsc2deficient cells, decreased ETV2 expression results in reduced Parpbp mRNA and PARPBP protein. Reduction in ETV2 or PARPBP protein levels induces ER stress, with increased phosphorylation of elF-2a and CHOP expression, resulting in increased apoptosis and cell death.

analyses of all endpoints were performed using one-way ANOVA, followed by a Tukey post hoc test or one- or two-tailed $t$ test. $P<$ 0.05 was considered statistically significant. Analyses were performed using GraphPad Prism 8.3 (GraphPad Software).

\section{Data Availability}

The microarray data from this publication have been deposited to the Gene Expression Omnibus database and assigned the identifier ID GSE183110 on the following link: https://www.ncbi.nlm.nih.gov/ geo $/$ query $/$ acc.cgi?acc=GSE183110.

\section{Supplementary Information}

Supplementary Information is available at https://doi.org/10.26508/lsa. 202201369

\section{Acknowledgements}

We are indebted to patients who participated in our protocols. This work was supported in part by the National Institutes of Health (U01-HL 131022 to S ElChemaly and T32HL007633-35 to J Ng) and the Division of Intramural
Research, National Institutes of Health, National Heart, Lung, and Blood Institute (J Moss) and the Anne Levine LAM Research Fund (S El-Chemaly).

\section{Author Contributions}

S Shrestha: conceptualization, data curation, formal analysis, investigation, methodology, and writing-original draft, review, and editing.

A Lamattina: data curation, formal analysis, investigation, and writing-review and editing.

G Pacheco-Rodriguez: conceptualization, data curation, investigation, and writing-review and editing.

J Ng: conceptualization, formal analysis, investigation, and writing-review and editing.

X Liu: data curation, investigation, and writing-review and editing. A Sonawane: formal analysis, investigation, methodology, and writing-review and editing.

J Imani: data curation, formal analysis, investigation, and writing-review and editing.

W Qiu: formal analysis, investigation, methodology, and writing-review and editing.

K Kosmas: data curation, formal analysis, investigation, and writing-review and editing.

P Louis: formal analysis, investigation, and writing-review and editing. 
A Hentschel: investigation and writing-review and editing. WK Steagall: conceptualization, data curation, formal analysis, investigation, and writing-review and editing.

R Onishi: data curation, formal analysis, and investigation.

$\mathrm{H}$ Christou: conceptualization, formal analysis, and writing-review and editing.

EP Henske: formal analysis, methodology, and writing-review and editing.

K Glass: conceptualization, formal analysis, investigation, methodology, and writing-review and editing.

MA Perrella: conceptualization, formal analysis, investigation, methodology, and writing-review and editing.

J Moss: conceptualization, formal analysis, supervision, funding acquisition, investigation, project administration, and writing-review and editing.

K Tantisira: conceptualization, formal analysis, methodology, and writing-review and editing.

S El-Chemaly: conceptualization, data curation, formal analysis, supervision, funding acquisition, investigation, methodology, project administration, and writing-original draft, review, and editing

\section{Conflict of Interest Statement}

The authors declare that they have no conflict of interest.

\section{References}

Abedin MJ, Nguyen A, Jiang N, Perry CE, Shelton JM, Watson DK, Ferdous A (2014) Fli1 acts downstream of Etv2 to govern cell survival and vascular homeostasis via positive autoregulation. Circ Res 114: 1690-1699. doi:10.1161/CIRCRESAHA.1134303145

Anderson P, Kedersha N (2002) Stressful initiations. J Cell Sci 115: 3227-3234. doi:10.1242/jcs.115.16.3227

Bailey TL, Boden M, Buske FA, Frith M, Grant CE, Clementi L, Ren J, Li WW, Noble WS (2009) MEME SUITE: Tools for motif discovery and searching. Nucleic Acids Res 37: W202-W208. doi:10.1093/nar/gkp335

Benjamini Y, Hochberg Y (1995) Controlling the false discovery rate: A practical and powerful approach to multiple testing. I R Stat Soc Series B Methodol 57: 289-300. doi:10.1111/j.2517-6161.1995.tb02031.x

Burkovics P, Dome L, Juhasz S, Altmannova V, Sebesta M, Pacesa M, Fugger K, Sorensen CS, Lee MY, Haracska L, et al (2016) The PCNA-associated protein PARI negatively regulates homologous recombination via the inhibition of DNA repair synthesis. Nucleic Acids Res 44: 3176-3189. doi:10.1093/nar/gkw024

Butler A, Hoffman P, Smibert P, Papalexi E, Satija R (2018) Integrating singlecell transcriptomic data across different conditions, technologies, and species. Nat Biotechnol 36: 411-420. doi:10.1038/nbt.4096

Carnevale J, Ross L, Puissant A, Banerji V, Stone RM, DeAngelo DJ, Ross KN, Stegmaier K (2013) SYK regulates mTOR signaling in AML. Leukemia 27: 2118-2128. doi:10.1038/leu.2013.89

Carsillo T, Astrinidis A, Henske EP (2000) Mutations in the tuberous sclerosis complex gene TSC2 are a cause of sporadic pulmonary lymphangioleiomyomatosis. Proc Natl Acad Sci U S A 97: 6085-6090. doi:10.1073/pnas.97.11.6085

Cheadle JP, Reeve MP, Sampson JR, Kwiatkowski DJ (2000) Molecular genetic advances in tuberous sclerosis. Hum Genet 107: 97-114. doi:10.1007/ s004390000348
Crino PB, Nathanson KL, Henske EP (2006) The tuberous sclerosis complex. N Engl J Med 355: 1345-1356. doi:10.1056/NEJMra055323

Cui Y, Steagall WK, Lamattina AM, Pacheco-Rodriguez G, Stylianou M, Kidambi P, Stump B, Golzarri F, Rosas IO, Priolo C, et al (2017) Aberrant SYK kinase signaling is essential for tumorigenesis induced by TSC2 inactivation. Cancer Res 77: 1492-1502. doi:10.1158/0008-5472.CAN-162755

Dai M, Wang P, Boyd AD, Kostov G, Athey B, Jones EG, Bunney WE, Myers RM, Speed TP, Akil H, et al (2005) Evolving gene/transcript definitions significantly alter the interpretation of GeneChip data. Nucleic Acids Res 33: e175. doi:10.1093/nar/gni179

Davis JA, Koenig AL, Lubert A, Chestnut B, Liu F, Palencia Desai S, Winkler T, Pociute K, Choi K, Sumanas S (2018) ETS transcription factor Etsrp/Etv2 is required for lymphangiogenesis and directly regulates vegfr3/flt 4 expression. Dev Biol 440: 40-52. doi:10.1016/j.ydbio.2018.05.003

Davis JM, Hyjek E, Husain AN, Shen L, Jones J, Schuger LA (2013) Lymphatic endothelial differentiation in pulmonary lymphangioleiomyomatosis cells. J Histochem Cytochem 61: 580-590. doi:10.1369/ 0022155413489311

Feng FY, Brenner JC, Hussain M, Chinnaiyan AM (2014) Molecular pathways: Targeting ETS gene fusions in cancer. Clin Cancer Res 20: 4442-4448. doi:10.1158/1078-0432.CCR-13-0275

Fruchon S, Kheirallah S, Al Saati T, Ysebaert L, Laurent C, Leseux L, Fournié J], Laurent G, Bezombes C (2012) Involvement of the Syk-mTOR pathway in follicular lymphoma cell invasion and angiogenesis. Leukemia 26 : 795-805. doi:10.1038/leu.2011.248

Fu R, Gillen AE, Sheridan RM, Tian C, Daya M, Hao Y, Hesselberth JR, Riemondy KA (2020) clustifyr: An R package for automated single-cell RNA sequencing cluster classification. F1000Res 9: 223. doi:10.12688/ f1000research.22969.2

Garry DJ (2016) Etv2 is a master regulator of hematoendothelial lineages. Trans Am Clin Climatol Assoc 127: 212-223.

Gautier L, Cope L, Bolstad BM, Irizarry RA (2004) affy: Analysis of Affymetrix GeneChip data at the probe level. Bioinformatics 20: 307-315. doi:10.1093/bioinformatics/btg405

Gentleman RC, Carey VJ, Bates DM, Bolstad B, Dettling M, Dudoit S, Ellis B, Gautier L, Ge Y, Gentry J, et al (2004) Bioconductor: Open software development for computational biology and bioinformatics. Genome Biol 5: R80. doi:10.1186/gb-2004-5-10-r80

Glasgow CG, Taveira-Dasilva AM, Darling TN, Moss J (2008) Lymphatic involvement in lymphangioleiomyomatosis. Ann N Y Acad Sci 1131: 206-214. doi:10.1196/annals.1413.018

Glass K, Huttenhower C, Quackenbush J, Yuan GC (2013) Passing messages between biological networks to refine predicted interactions. PLOS One 8: e64832. doi:10.1371/journal.pone.0064832

Goncharova EA, Goncharov DA, Eszterhas A, Hunter DS, Glassberg MK, Yeung RS, Walker CL, Noonan D, Kwiatkowski DJ, Chou MM, et al (2002) Tuberin regulates p70 S6 kinase activation and ribosomal protein $\mathrm{S} 6$ phosphorylation. A role for the TSC2 tumor suppressor gene in pulmonary lymphangioleiomyomatosis (LAM). J Biol Chem 277: 30958-30967. doi:10.1074/jbc.M202678200

Grant CE, Bailey TL, Noble WS (2011) FIMO: Scanning for occurrences of a given motif. Bioinformatics 27: 1017-1018. doi:10.1093/bioinformatics/btr064

Guo M, Yu JJ, Perl AK, Wikenheiser-Brokamp KA, Riccetti M, Zhang EY, Sudha P, Adam M, Potter A, Kopras EJ, et al (2020) Single-cell transcriptomic analysis identifies a unique pulmonary lymphangioleiomyomatosis cell. Am J Respir Crit Care Med 202: 1373-1387. doi:10.1164/ rccm.201912$24450 C$

Habermann AC, Gutierrez AJ, Bui LT, Yahn SL, Winters NI, Calvi CL, Peter L, Chung M-I, Taylor CJ, Jetter C, et al (2020) Single-cell RNA sequencing reveals profibrotic roles of distinct epithelial and mesenchymal lineages in pulmonary fibrosis. Sci Adv 6: eaba1972. doi:10.1126/ sciadv.aba1972 
Huang DW, Sherman BT, Lempicki RA (2009a) Bioinformatics enrichment tools: Paths toward the comprehensive functional analysis of large gene lists. Nucleic Acids Res 37: 1-13. doi:10.1093/nar/gkn923

Huang DW, Sherman BT, Lempicki RA (2009b) Systematic and integrative analysis of large gene lists using DAVID bioinformatics resources. Nat Protoc 4: 44-57. doi:10.1038/nprot.2008.211

Irizarry RA, Hobbs B, Collin F, Beazer-Barclay YD, Antonellis KJ, Scherf U, Speed TP (2003) Exploration, normalization, and summaries of high density oligonucleotide array probe level data. Biostatistics 4: 249-264. doi:10.1093/biostatistics/4.2.249

Johnson SR, Taveira-DaSilva AM, Moss J (2016) Lymphangioleiomyomatosis. Clin Chest Med 37: 389-403. doi:10.1016/j.ccm.2016.04.002

Kabir AU, Lee TJ, Pan H, Berry JC, Krchma K, Wu J, Liu F, Kang HK, Hinman K, Yang $L$, et al (2018) Requisite endothelial reactivation and effective siRNA nanoparticle targeting of Etv2/Er71 in tumor angiogenesis. JCI Insight 3: e97349. doi:10.1172/jci.insight.97349

Kang YJ, Lu MK, Guan KL (2011) The TSC1 and TSC2 tumor suppressors are required for proper ER stress response and protect cells from ER stress-induced apoptosis. Cell Death Differ 18: 133-144. doi:10.1038/ cdd.2010.82

Kim JY, Lee DH, Kim JK, Choi HS, Dwivedi B, Rupji M, Kowalski J, Green SJ, Song H, Park WJ, et al (2019) ETV2/ER71 regulates the generation of FLK1+ cells from mouse embryonic stem cells through miR-126-MAPK signaling. Stem Cell Res Ther 10: 328. doi:10.1186/s13287-019-1466-8

Korsunsky I, Millard N, Fan J, Slowikowski K, Zhang F, Wei K, Brennar M, Loh P-R, Raychaudhuri S (2019) Fast, sensitive and accurate integration of single-cell data with Harmony. Nat Methods 16: 1289-1296. doi:10.1038/s41592-019-0619-0

Kosmas K, Filippakis H, Khabibullin D, Turkiewicz M, Lam HC, Yu J, Kedersha $\mathrm{NL}$, Anderson PJ, Henske EP (2021) TSC2 interacts with HDLBP/vigilin and regulates stress granule formation. Mol Cancer Res 19: 1389-1397. doi:10.1158/1541-7786.mcr-20-1046

Lee DH, Kim TM, Kim JK, Park C (2019) ETV2/ER71 transcription factor as a therapeutic vehicle for cardiovascular disease. Theranostics 9: 5694-5705. doi:10.7150/thno.35300

Leseux L, Hamdi SM, Al Saati T, Capilla F, Recher C, Laurent G, Bezombes C (2006) Syk-dependent mTOR activation in follicular lymphoma cells. Blood 108: 4156-4162. doi:10.1182/blood-2006-05-026203

Li J, Sidell N (2005) Growth-related oncogene produced in human breast cancer cells and regulated by Syk protein-tyrosine kinase. Int J Cancer 117: 14-20. doi:10.1002/ijc.21074

Li X, Moon G, Shin S, Zhang B, Janknecht R (2018) Cooperation between ETS variant 2 and Jumonji domain-containing 2 histone demethylases. Mol Med Rep 17: 5518-5527. doi:10.3892/mmr.2018.8507

Linderman GC, Zhao J, Roulis M, Bielecki P, Flavell RA, Nadler B, Kluger Y (2022) Zero-preserving imputation of single-cell RNA-seq data. Nat Commun 13: 192. doi:10.1038/s41467-021-27729-z

Liu F, Li D, Yu YY, Kang I, Cha MJ, Kim JY, Park C, Watson DK, Wang T, Choi K (2015) Induction of hematopoietic and endothelial cell program orchestrated by ETS transcription factor ER71/ETV2. EMBO Rep 16: 654-669. doi:10.15252/embr.201439939

Malyuchenko NV, Kotova EY, Kulaeva OI, Kirpichnikov MP, Studitskiy VM (2015) PARP1 inhibitors: Antitumor drug design. Acta Naturae 7: 27-37. doi:10.32607/20758251-2015-7-3-27-37

McCormack FX, Inoue Y, Moss J, Singer LG, Strange C, Nakata K, Barker AF, Chapman JT, Brantly ML, Stocks JM, et al (2011) Efficacy and safety of sirolimus in lymphangioleiomyomatosis. N Engl J Med 364: 1595-1606. doi:10.1056/NEJMoa1100391

NCBI Resource Coordinators (2013) Database resources of the national center for biotechnology information. Nucleic Acids Res 41: D8-D20. doi:10.1093/nar/gks1189
Nicolae CM, O'Connor MJ, Schleicher EM, Song C, Gowda R, Robertson G, Dovat S, Moldovan GL (2019) PARI (PARPBP) suppresses replication stressinduced myeloid differentiation in leukemia cells. Oncogene 38: 5530-5540. doi:10.1038/s41388-019-0810-x

O'Connor KW, Dejsuphong D, Park E, Nicolae CM, Kimmelman AC, D'Andrea AD, Moldovan GL (2013) PARI overexpression promotes genomic instability and pancreatic tumorigenesis. Cancer Res 73: 2529-2539. doi:10.1158/0008-5472.CAN-12-3313

Oliver G, Srinivasan RS (2010) Endothelial cell plasticity: How to become and remain a lymphatic endothelial cell. Development 137: 363-372. doi:10.1242/ dev.035360

Ozcan U, Ozcan L, Yilmaz E, Düvel K, Sahin M, Manning BD, Hotamisligil GS (2008) Loss of the tuberous sclerosis complex tumor suppressors triggers the unfolded protein response to regulate insulin signaling and apoptosis. Mol Cell 29: 541-551. doi:10.1016/j.molcel.2007.12.023

Pacheco-Rodriguez G, Steagall WK, Crooks DM, Stevens LA, Hashimoto H, Li S, Wang JA, Darling TN, Moss J (2007) TSC2 loss in lymphangioleiomyomatosis cells correlated with expression of CD44v6, a molecular determinant of metastasis. Cancer Res 67: 10573-10581. doi:10.1158/0008-5472.CAN-07-1356

Park S, Jang JW, Moon EY (2018) Spleen tyrosine kinase-dependent Nrf2 activation regulates oxidative stress-induced cell death in WiL2-NS human B lymphoblasts. Free Radic Res 52: 977-987. doi:10.1080/ 10715762.2018.1505044

Piao L, Nakagawa H, Ueda K, Chung S, Kashiwaya K, Eguchi H, Ohigashi H, Ishikawa O, Daigo Y, Matsuda K, et al (2011) C12orf48, termed PARP-1 binding protein, enhances poly(ADP-ribose) polymerase-1 (PARP-1) activity and protects pancreatic cancer cells from DNA damage. Genes Chromosomes Cancer 50: 13-24. doi:10.1002/gcc.20828

Rosset C, Netto CBO, Ashton-Prolla P (2017) TSC1 and TSC2 gene mutations and their implications for treatment in tuberous sclerosis complex: A review. Genet Mol Biol 40: 69-79. doi:10.1590/1678-4685-GMB-20150321

Seyama K, Kumasaka T, Souma S, Sato T, Kurihara M, Mitani K, Tominaga S, Fukuchi Y (2006) Vascular endothelial growth factor-D is increased in serum of patients with lymphangioleiomyomatosis. Lymphat Res Biol 4: 143-152. doi:10.1089//rb.2006.4.143

Singh BN, Gong W, Das S, Theisen JWM, Sierra-Pagan JE, Yannopoulos D, Skie E, Shah P, Garry MG, Garry DJ (2019) Etv2 transcriptionally regulates Yes1 and promotes cell proliferation during embryogenesis. Sci Rep 9: 9736. doi:10.1038/s41598-019-45841-5

Steagall WK, Pacheco-Rodriguez G, Glasgow CG, Ikeda Y, Lin JP, Zheng G, Moss J (2013) Osteoprotegerin contributes to the metastatic potential of cells with a dysfunctional TSC2 tumor-suppressor gene. Am J Pathol 183: 938-950. doi:10.1016/j.ajpath.2013.05.024

Uckun FM, Ma H, Zhang J, Ozer Z, Dovat S, Mao C, Ishkhanian R, Goodman P, Qazi S (2012) Serine phosphorylation by SYK is critical for nuclear localization and transcription factor function of Ikaros. Proc Natl Acad Sci U S A 109: 18072-18077. doi:10.1073/pnas.1209828109

Uhlén M, Fagerberg L, Hallström BM, Lindskog C, Oksvold P, Mardinoglu A, Sivertsson A, Kampf C, Sjöstedt E, Asplund A, et al (2015) Proteomics. Tissue-based map of the human proteome. Science 347: 1260419. doi:10.1126/science.1260419

Varisli L (2013) Meta-analysis of the cell cycle related C12orf48. Biocell 37: 11-16. doi:10.32604/biocell.2013.37.011

Walker JA, Hughes DA, Hedges DJ, Anders BA, Laborde ME, Shewale J, Sinha SK, Batzer MA (2004) Quantitative PCR for DNA identification based on genome-specific interspersed repetitive elements. Genomics 83: 518-527. doi:10.1016/j.ygeno.2003.09.003

Wei GH, Badis G, Berger MF, Kivioja T, Palin K, Enge M, Bonke M, Jolma A, Varjosalo M, Gehrke AR, et al (2010) Genome-wide analysis of ETSfamily DNA-binding in vitro and in vivo. EMBO I 29: 2147-2160. doi:10.1038/emboj.2010.106 
Life Science Alliance

Yamamizu K, Matsunaga T, Katayama S, Kataoka H, Takayama N, Eto K, Nishikawa S, Yamashita JK (2012) PKA/CREB signaling triggers initiation of endothelial and hematopoietic cell differentiation via Etv2 induction. Stem Cells 30: 687-696. doi:10.1002/stem.1041

Yao J, Taveira-DaSilva AM, Jones AM, Julien-Williams P, Stylianou M, Moss J (2014) Sustained effects of sirolimus on lung function and cystic lung lesions in lymphangioleiomyomatosis. Am J Respir Crit Care Med 190: 1273-1282. doi:10.1164/rccm.201405-09180C

Young LR, Vandyke R, Gulleman PM, Inoue Y, Brown KK, Schmidt LS, Linehan WM, Hajjar F, Kinder BW, Trapnell BC, et al (2010) Serum vascular endothelial growth factor-D prospectively distinguishes lymphangioleiomyomatosis from other diseases. Chest 138: 674-681. doi:10.1378/chest.10-0573

Yu B, Ding Y, Liao X, Wang C, Wang B, Chen X (2019) Overexpression of PARPBP correlates with tumor progression and poor prognosis in hepatocellular carcinoma. Dig Dis Sci 64: 2878-2892. doi:10.1007/ s10620-019-05608-4

Yu JJ, Robb VA, Morrison TA, Ariazi EA, Karbowniczek M, Astrinidis A, Wang C, Hernandez-Cuebas L, Seeholzer LF, Nicolas E, et al (2009) Estrogen promotes the survival and pulmonary metastasis of tuberin-null cells. Proc Natl Acad Sci U S A 106: 2635-2640. doi:10.1073/ pnas.0810790106

Yu S, Huang H, Iliuk A, Wang WH, Jayasundera KB, Tao WA, Post CB, Geahlen RL (2013) Syk inhibits the activity of protein kinase A by phosphorylating tyrosine 330 of the catalytic subunit. J Biol Chem 288: 10870-10881. doi:10.1074/jbc.M112.426130

Yue M, Pacheco G, Cheng T, Li J, Wang Y, Henske EP, Schuger L (2016) Evidence supporting a lymphatic endothelium origin for angiomyolipoma, a TSC2(-) tumor related to lymphangioleiomyomatosis. Am J Pathol 186: 1825-1836. doi:10.1016/j.ajpath.2016.03.009

Zhao C, Gomez GA, Zhao Y, Yang Y, Cao D, Lu J, Yang H, Lin S (2018) ETV2 mediates endothelial transdifferentiation of glioblastoma. Signal Transduct Target Ther 3: 4. doi:10.1038/s41392-018-0007-8

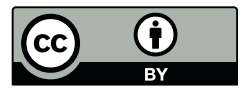

License: This article is available under a Creative Commons License (Attribution 4.0 International, as described at https://creativecommons.org/ licenses/by/4.0/). 